\title{
Quadrature Rules for Finite Element Approximations of 1D Nonlocal Problems
}

\author{
Xiaoping Zhang ${ }^{\mathrm{a}}$, Max Gunzburger ${ }^{\mathrm{b}}$, Lili $\mathrm{Ju}^{\mathrm{c}}$ \\ ${ }^{a}$ School of Mathematics and Statistics, Wuhan University, Wuhan, Hubei 430072, China. \\ ${ }^{b}$ Department of Scientific Computing, Florida State University, Tallahassee, FL 32306, USA. \\ ${ }^{c}$ Department of Mathematics, University of South Carolina, Columbia, SC 29208, USA.
}

\begin{abstract}
It is well known that calculations of the entries of the stiffness matrix in the finite element approximations of nonlocal diffusion and mechanics models are often very time-consuming due to the double integration process over the domain and the singularities of the nonlocal kernel functions. In this paper, we propose some effective and accurate quadrature rules for computing these double integrals for one-dimensional nonlocal problems; in particular, for problems with highly singular kernels, the corresponding inner integrals can be first evaluated exactly in our method, and the outer one then will be approximated by some popular quadrature rules. With these quadrature rules, the assembly of the stiffness matrix in the finite element method for the nonlocal problems becomes similar to that for the classical partial differential equations and is thus quite efficient.
\end{abstract}

Keywords: Nonlocal problems, finite element approximations, finite-part integrals, product rules, DE rules

2010 MSC: 65R20, 65N30, 82C21, 41A55, 45B05, 44A35

\section{Introduction}

Nonlocal theories for continuum mechanics and diffusion have been of relatively recent and growing interest. For example, peridynamics (PD) models, proposed in $[1,2,3]$, are integrodifferential nonlocal continuum model that provides an alternative to classic continuum mechanics which is based on partial differential equations (PDEs). PD models do not involve spatial derivatives; their effectiveness for modeling singular behavior in solids has been demonstrated through numerical simulations of crack nucleation and growth, fracture and failure of composites, polycrystals, and nanofiber networks. The peridynamic model has been applied in several settings; see, e.g. $[1,2,3,4,5,6,7,8]$. Modeling and theoretical studies regarding peridynamics are found in, e.g., $[9,10]$ whereas its computational solution is considered in, e.g., [11, 12, 13, 14, 15, 16]. Another setting where nonlocal models are becoming increasingly popular is in the modeling of anomalous diffusion (AD) [9]. The spatial operators in the PD and anomalous superdiffusion settings share

\footnotetext{
${ }^{\star 2}$ X. Zhang's research is partially supported by National Natural Science Foundation of China under No. 11101317 and 91330107, M. Gunzburger's research is partially supported by US National Science Foundation under grant number DMS-1315259, L. Ju's research is partially supported by US National Science Foundation under grant numbers DMS-1215659 and DMS-1521965.

Email addresses: xpzhang.math@whu.edu.cn (Xiaoping Zhang), mgunzburger@fsu.edu (Max Gunzburger), ju@math.sc.edu (Lili Ju)
} 
many similarities and thus make the study one model relevant to the other. A common feature of the $\mathrm{PD}$ and $\mathrm{AD}$ models considered here is the introduction of a horizon parameter $\delta$ that characterizes the range of nonlocal interactions $[10,17]$. As $\delta \rightarrow 0$, nonlocal effects diminish and the limit provides natural links between the nonlocal models and the classical (local) PDE-based models in cases for which the latter are well defined.

Several discretization methods have been proposed for the spatial discretization of PD and AD nonlocal models, including finite difference methods (FD) [14], finite element methods (FE) [12, 14, $18,19]$, collocation methods [20, 21], and so on. In the steady-state setting, PD and AD models are integral equations, and thus collocation methods are frequently used [19] due to the simplicity of their implementation. Unfortunately, the accuracy of collocation methods is often unsatisfactory compared with finite element methods under the same grid resolution. Galerkin finite element discretizations, being based on variational formulations, has been widely investigated for integral equation as in boundary element methods; for example, see [22]. They require a double integration process; seemingly, treating double integrals is difficult to do efficiently and accurately due to the singularity of the kernel functions appearing in the integrand. In [22], the authors introduce suitable variable transformations that render the singular integrand analytic, which permits the numerical approximation of the integral by means of standard quadrature methods. If a hypersingular kernel function is encountered, regularization by means of integration by parts is first applied and the resulting process can be effected. In quantitative finance, a class of price processes which follows Lévy processes, can be shown to obey a partial integro-differential equation (PIDE); for example, see [23] in which Galerkin finite element approximations are also discussed for PIDE, and the entries of stiffness matrix are computed by means of repeated integration by parts, assuming that the $i$-th antiderivative of the singular Lévy density is explicitly available. As for PD and AD models, an approach for the evaluation of the double integral was proposed in $[12,24]$; the inner integral is first split into the sum of many integrals to avoid the singularity, and then certain quadrature rules are used to approximate the various outer and inner integrals. Note that the outer integral must be treated carefully due to end-point singularities; for example, a high-order global adaptive GaussKronrod quadrature formula was used in [24] to compute the outer integral. Such approaches can require thousands of quadrature points to evaluate each entry in the finite element stiffness matrix, resulting in a very expensive assembly process, especially in two and three dimensions.

In this paper, we develop a novel and efficient way to assemble the stiffness matrix for Galerkin finite element discretizations of one-dimensional steady-state PD and AD models. Inspired by the work in [25], we obtain some basic quadrature rules with explicit expression to compute the double integrals having different singularities that appear in the entries of the stiffness matrix. Once having these rules, the computation of the singular double integrals can be done in a similar way to that in [25], i.e., the inner integrals can be evaluated exactly by the proposed product rules, and the outer ones can be evaluated by a double exponential rule. However, due to the effect of the horizon parameter $\delta$, this process is more difficult, and thus should be treated more carefully. Such an approach can greatly improve the accuracy and efficiency of the assembly of the stiffness matrix. We remark that our work is a direct one without regularization and is thus different from [22, 23].

The rest of the paper is organized as follows. In Section 1.1, we first describe the model 1D nonlocal problem we consider. Finite-part integrals, including their definitions and variable transformations, are introduced and discussed in Section 2. Some quadrature rules are derived in Section 3 and their application to the evaluation of the entries of the stiffness matrix in finite element discretization for nonlocal problems is carefully investigated in Sections 4 and 5. Numerical 
experiments are presented in Section 6 to illustrate the efficiency and accuracy of the proposed method. Finally, concluding remark are provided in Section 7.

\subsection{The $1 D$ nonlocal problem}

We consider the Dirichlet constrained-value problem associated with a one-dimensional nonlocal linear model. Without loss of generality, we choose the domain of interest $\Omega=(0,1)$. A nonlocal operator $\mathcal{L}_{\delta}$ is defined as, for any function $u=u(x): \Omega \rightarrow \mathbb{R}$,

$$
\mathcal{L}_{\delta} u(x)=\frac{2-2 s}{\delta^{2-2 s}} \int_{B_{\delta}(x)} \frac{u(y)-u(x)}{|y-x|^{1+2 s}} d y, \quad \forall x \in \Omega,
$$

where $s \in[0,1)$, the constant $\delta>0$ is referred to as the horizon, and $B_{\delta}(x)=\{y \in \mathbb{R}:|y-x|<\delta\}$ denotes the interval centered at $x$ having length $2 \delta$. We define the interaction domain as $\Omega_{\mathcal{I}}=$ $\{y \in \mathbb{R} \backslash \Omega:|y-x|<\delta$ for $x \in \Omega\}=(-\delta, 0] \cup[1,1+\delta)$, i.e., $\Omega_{\mathcal{I}}$ is the set of points exterior to $\Omega$ that interact with points within $\Omega$. We then define the nonlocal problem, constrained on a volume having nonzero measure, as

$$
\begin{cases}-\mathcal{L}_{\delta} u=f(x) & \text { in } \Omega, \\ u(x)=g(x) & \text { on } \Omega_{\mathcal{I}},\end{cases}
$$

where $f(x)$ and $g(x)$ are given functions. For $s=0,(2)$ is a one-dimensional peridynamics model [1], whereas for $s \in(0,1)$, it is a one-dimensional model for superdiffussion [9]. Note that, in the nonlocal setting, well posedness requires [9] a constraint acting on a volume with positive measure (the second equation in (2)) in contrast with the local PDE setting for which constraints are applied on the boundary of the domain. Note that for sufficiently smooth $u$, as $\delta \rightarrow 0$, the problem (2) reduces to the Poisson problem [9] whereas, as $\delta \rightarrow \infty$, it reduces to the fractional Laplacian problem involving $(-\Delta)^{s}$ posed on a bounded domain [24].

A weak formulation of (2) is given by

$$
-\frac{2-2 s}{\delta^{2-2 s}} \int_{\Omega} \int_{\Omega^{\prime} \cap B_{\delta}(x)} \frac{u(y)-u(x)}{|y-x|^{1+2 s}} v(x) d y d x=\int_{\Omega} f v d x,
$$

where $\Omega^{\prime}=\Omega \cup \Omega_{\mathcal{I}}$ and the solution $u$ and the test functions $v$ belong to appropriate function spaces. An alternative weak formulation of (2) is given by

$$
\frac{1-s}{\delta^{2-2 s}} \int_{\Omega} \int_{\Omega^{\prime} \cap B_{\delta}(x)} \frac{u(y)-u(x)}{|y-x|^{1+2 s}}(v(y)-v(x)) d y d x=\int_{\Omega} f v d x .
$$

In the function space setting, the forms (3) and (4) are equivalent. For $s=0$, standard finite element methods based on (3) and (4) were investigated in [12] and [14, 24], respectively. We extend the implementation of finite element methods to $0 \leq s<1$ based on the formulation (3), using a completely different approach than that taken in [12].

\section{Hadamard finite-part integrals}

In this section, we briefly review the concept of Hadamard finite-part integrals. Consider the integral of the form

$$
\int_{a}^{b} \frac{w(y)}{|y-x|^{1+2 s}} d y, \quad x \in(a, b)
$$


If $s \geq 0$, the integrand is in general not integrable in the classic Riemann sense so that (5) should be understood in the Hadamard finite-part sense. There are several mathematically equivalent definitions for Hadamard finite-part integrals; we adopt a definition proposed in [26]. Consider the divergent integral

$$
f_{a}^{b} \sigma(y-x) w(y) d y
$$

satisfying the following assumptions:

1. the kernel $\sigma(z)$ is a real-valued function which is continuous on $[a-b, b-a] \backslash\{0\}$ and $|\sigma(z)| \leq$ $C|z|^{-\lambda}$ holds for some $1 \leq \lambda \in \mathbb{R}$

2. the $(m-1)$-th derivative of $w(y)$ is a Hölder function of degree $\alpha$ with $m \in \mathbb{N}, 0<\alpha \leq 1$, and $m+\alpha>\lambda$, i.e., $w \in C^{m-1, \alpha}(a, b)$.

Definition 1. Under the above assumptions, the Hadamard finite-part integral (6) is defined as

$$
f_{a}^{b} \sigma(y-x) w(y) d y=\lim _{\epsilon \rightarrow 0}\left(\int_{\Omega_{\epsilon}} \sigma(y-x) w(y) d y+\sum_{j=0}^{m-1} \frac{w^{(j)}(x)}{j !}\left[\sigma_{j}(\epsilon)-\sigma_{j}(-\epsilon)\right]\right)
$$

where $\Omega_{\epsilon}=(a, b) \backslash(x-\epsilon, x+\epsilon)$ and $\sigma_{j}(z)$ denotes the antiderivative of the function $z^{j} \sigma(z)$, i.e.,

$$
\sigma_{j}^{\prime}(z)=z^{j} \sigma(z), \quad z \in[a-b, b-a] \backslash\{0\}, \quad j=0, \cdots, m-1 .
$$

With $\sigma(y-x)=|y-x|^{-1-2 s}$, we then have that the integral (5) can be defined, for $x \in(a, b)$, as

$$
f_{a}^{b} \frac{w(y)}{|y-x|^{1+2 s}} d y= \begin{cases}\lim _{\epsilon \rightarrow 0}\left(\int_{\Omega_{\epsilon}} \frac{w(y)}{|y-x|} d y+2 w(x) \ln \epsilon\right) & \text { for } s=0 \\ \lim _{\epsilon \rightarrow 0}\left(\int_{\Omega_{\epsilon}} \frac{w(x)}{|y-x|^{1+2 s}} d y-\frac{w(x)}{s \epsilon^{2 s}}\right) & \text { for } s \in(0,1) .\end{cases}
$$

In particular, by setting $w(x)=1$, we obtain

$$
f_{a}^{b} \frac{1}{|y-x|^{1+2 s}} d y= \begin{cases}\ln |x-a|+\ln |b-x| & \text { for } s=0 \\ -\frac{1}{2 s}\left((x-a)^{-2 s}+(b-x)^{-2 s}\right) & \text { for } s \in(0,1) .\end{cases}
$$

Remark 2. It can be seen from (7) that there exists an interesting phenomenon associated with the finite-part integral. Consider the example of $a=-1, b=1, x=0$, and $s=1 / 2$ in (7); we then have

$$
f_{-1}^{1} \frac{1}{y^{2}} d y=-2
$$

This is at odds with the fact that the value of a Riemann integral for a positive function is always positive; this is a remarkable difference between Riemann integrals and Hadamard finite-part integrals. 
Definition 3. If $w$ has less regularity, a more general definition of (6) is

$$
\begin{aligned}
f_{a}^{b} \sigma(y-x) w(y) d y=\lim _{\epsilon \rightarrow 0} & \left(\int_{\Omega_{\epsilon}^{+}} \sigma(y-x) w(y) d y+\sum_{j=0}^{m-1} \frac{\sigma_{j}(\epsilon) w^{(j)}(x+)}{j !}\right. \\
& \left.+\int_{\Omega_{\epsilon}^{-}} \sigma(y-x) w(y) d y-\sum_{j=0}^{m-1} \frac{\sigma_{j}(-\epsilon) w^{(j)}(x-)}{j !}\right),
\end{aligned}
$$

where $\Omega_{\epsilon}^{-}=(a, x-\epsilon), \Omega_{\epsilon}^{+}=(x+\epsilon, b), w^{(j)}(x+)$ and $w^{(j)}(x-)$ are the right and left $j$-th order derivatives of $w$. Moreover,

$$
f_{a}^{b} \sigma(y-a) w(y) d y=\lim _{\epsilon \rightarrow 0}\left(\int_{a+\epsilon}^{b} \sigma(y-a) w(y) d y+\sum_{j=0}^{m-1} \frac{\sigma_{j}(\epsilon) w^{(j)}(a+)}{j !}\right)
$$

and

$$
f_{a}^{b} \sigma(y-b) w(y) d y=\lim _{\epsilon \rightarrow 0}\left(\int_{a}^{b-\epsilon} \sigma(y-b) w(y) d y-\sum_{j=0}^{m-1} \frac{\sigma_{j}(-\epsilon) w^{(j)}(b-)}{j !}\right) .
$$

The definition (8) is often used to design nodal-type quadrature rules for evaluating (6) (see $[21,27])$, and (9) and (10) can be used to construct quadrature rules for computing the divergent integrals with an endpoint singularity. With $\sigma(y-a)=(y-a)^{-2 s}$ and $\sigma(y-b)=(b-y)^{-2 s}$, we then have

$$
f_{a}^{b} \frac{w(y)}{(y-a)^{2 s}} d y= \begin{cases}\lim _{\epsilon \rightarrow 0}\left(\int_{a+\epsilon}^{b} \frac{w(y)}{y-a} d y+w(a) \ln \epsilon\right) & \text { for } s=\frac{1}{2} \\ \lim _{\epsilon \rightarrow 0}\left(\int_{a+\epsilon}^{b} \frac{w(y)}{(y-a)^{2 s}} d y+w(a) \frac{\epsilon^{1-2 s}}{1-2 s}\right) & \text { for } s \in\left(\frac{1}{2}, 1\right)\end{cases}
$$

and

$$
f_{a}^{b} \frac{w(y)}{(b-y)^{2 s}} d y= \begin{cases}\lim _{\epsilon \rightarrow 0}\left(\int_{a}^{b-\epsilon} \frac{w(y)}{b-y} d y+w(b) \ln \epsilon\right) & \text { for } s=\frac{1}{2} \\ \lim _{\epsilon \rightarrow 0}\left(\int_{a+\epsilon}^{b} \frac{w(y)}{(b-y)^{2 s}} d y+w(b) \frac{\epsilon^{1-2 s}}{1-2 s}\right) & \text { for } s \in\left(\frac{1}{2}, 1\right) .\end{cases}
$$

Remark 4. If $0<s<\frac{1}{2}$, the two integrals above are only weakly singular at the endpoints.

Another remarkable difference between Hadamard finite-part integrals and Riemann integrals exists in the transformation of integration variables. In the following, we list some concise relations which are used in this paper, and refer to [26] for details. Let

$$
y=\frac{b-a}{\beta-\alpha} t+\frac{a \beta-b \alpha}{\beta-\alpha}, \quad x=\frac{b-a}{\beta-\alpha} \tau+\frac{a \beta-b \alpha}{\beta-\alpha}, \quad p(t)=w\left(\frac{b-a}{\beta-\alpha} t+\frac{a \beta-b \alpha}{\beta-\alpha}\right) .
$$


Then,

$$
\begin{aligned}
& f_{a}^{b} \frac{w(y)}{|y-x|^{1+2 s}} d y= \begin{cases}f_{\alpha}^{\beta} \frac{p(t)}{|t-\tau|} d t+2 p(s) \ln \frac{b-a}{\beta-\alpha} & \text { for } s=0 \\
\left(\frac{b-a}{\beta-\alpha}\right)^{-2 s} f_{\alpha}^{\beta} \frac{p(t)}{|t-\tau|^{1+2 s}} d t & \text { for } s \in(0,1)\end{cases} \\
& f_{a}^{b} \frac{w(y)}{(y-a)^{2 s}} d y= \begin{cases}f_{\alpha}^{\beta} \frac{p(t)}{t-\alpha} d t+p(\alpha) \ln \frac{b-a}{\beta-\alpha} & \text { for } s=\frac{1}{2} \\
\left(\frac{b-a}{\beta-\alpha}\right)^{1-2 s} f_{\alpha}^{\beta} \frac{p(t)}{(t-\alpha)^{2 s}} d t & \text { for } s \in\left(\frac{1}{2}, 1\right)\end{cases} \\
& f_{a}^{b} \frac{w(y)}{(b-y)^{2 s}} d y= \begin{cases}f_{\alpha}^{\beta} \frac{p(t)}{\beta-t} d t+p(\beta) \ln \frac{b-a}{\beta-\alpha} \\
\left(\frac{b-a}{\beta-\alpha}\right)^{1-2 s} f_{\alpha}^{\beta} \frac{p(t)}{(\beta-t)^{2 s}} d t & \text { for } s \in\left(\frac{1}{2}, 1\right) .\end{cases}
\end{aligned}
$$

As an example but without loss of generality, we only provide a derivation of (11) with $s=0$ :

$$
\begin{aligned}
f_{a}^{b} \frac{w(y)}{|y-x|} d y & =\lim _{\epsilon \rightarrow 0}\left(\left(\int_{a}^{x-\epsilon}+\int_{x+\epsilon}^{b}\right) \frac{w(y)}{|y-x|} d y+2 w(x) \ln \epsilon\right) \\
& =\lim _{\epsilon \rightarrow 0}\left(\left(\int_{\alpha}^{s-\frac{\beta-\alpha}{b-a} \epsilon}+\int_{s+\frac{\beta-\alpha}{b-a} \epsilon}^{\beta}\right) \frac{p(t)}{|t-s|} d t+2 p(s) \ln \epsilon\right) \\
& =\lim _{\epsilon \rightarrow 0}\left(\left(\int_{\alpha}^{s-\delta}+\int_{s+\delta}^{\beta}\right) \frac{p(t)}{|t-s|} d t+2 p(s) \ln \frac{b-a}{\beta-\alpha} \delta\right) \\
& =f_{\alpha}^{\beta} \frac{p(t)}{|t-s|} d t+2 p(s) \ln \frac{b-a}{\beta-\alpha},
\end{aligned}
$$

where $\delta=\frac{\beta-\alpha}{b-a} \epsilon$ has been used in the third step.

\section{Basic quadrature rules}

Numerous research effort has been devoted to numerical evaluations of singular integrals, such as Gaussian-type methods [28, 29, 30, 31], Newton-Cotes type methods [27, 32], transformation methods [33, 34], and some other methods [35]. Especially, Gaussian-type methods are often used to compute the stiffness matrix in finite element discretization of singular integral equations. However, most of these works are limited to hypersingular integrals with singularity of integer order. In this section, we discuss the design of some basic quadrature rules for computing hypersingular integrals with singularity of fractional order and weakly singular integrals with endpoint singularities on the standard interval $(-1,1)$ that will be used in the finite element discretization of the nonlocal problem (2).

\subsection{Product rule}

The idea of the product rule originated in [25]. We begin the discussion with two results. 
Lemma 5. Let $K(x, y)$ denote an integration kernel that may have a hyper-singularity in $(-1,1)$ or at the endpoints, or is nearly singular at the endpoints. Then, we have the approximation

$$
\int_{-1}^{1} K(x, y) w(y) d y \approx \sum_{k=1}^{n} \omega_{k}(x) w\left(x_{k}\right),
$$

where $\left\{x_{k}\right\}_{k=1}^{n}$ denote the zeros of the Legendre polynomials $P_{n}(x)$ of degree $n$ and

$$
\omega_{k}(x)=\frac{1}{2} \lambda_{k} \sum_{l=0}^{n-1}(2 l+1) P_{l}\left(x_{k}\right) \mu_{l}(x)
$$

with $\lambda_{k}$ denoting the classical Christoffel numbers [36, p. 47-48] associated with the n-point GaussLegendre formula $\int_{-1}^{1} v(x) d x \approx \sum_{k=1}^{n} \lambda_{k} v\left(x_{k}\right)$ and $\mu_{l}(x)$ denoting the modified moment of the kernel $K(x, y)$ given by $\mu_{l}(x)=\int_{-1}^{1} K(x, y) P_{l}(y) d y$.

Proof. The proof can be found in [37]. For completeness, we still provide a simple proof. Let $\Pi_{k} w(x)$ denote the Lagrange interpolant of $w(x)$ with respect to the points $\left\{x_{k}\right\}_{k=1}^{n}$, i.e., $\Pi_{k} w(x)=$ $\sum_{k=1}^{n} w\left(x_{k}\right) \ell_{k}(x)$ with $\ell_{k}(x)=\prod_{\substack{j=1 \\ j \neq k}}^{n} \frac{x-x_{j}}{x_{k}-x_{j}}$ which is a polynomial of degree $n-1$. Replacing $w(x)$ in (14) by $\Pi_{k} w(x)$ leads to

$$
\int_{-1}^{1} K(x, y) w(y) d y \approx \sum_{k=1}^{n} w\left(x_{k}\right) \int_{-1}^{1} K(x, y) \ell_{k}(y) d y
$$

which implies that

$$
\omega_{k}(x)=\int_{-1}^{1} K(x, y) \ell_{k}(y) d y .
$$

By using the orthogonality property of Legendre polynomials and the fact that $\ell_{k}(y) P_{l}(y)$ is a polynomial of degree less than $2 n$, we see that

$$
\ell_{k}(y)=\sum_{l=0}^{n-1} a_{l}^{(k)} P_{l}(y)
$$

with

$$
a_{l}^{(k)}=\frac{2 l+1}{2} \int_{-1}^{1} \ell_{k}(x) P_{l}(x) d x=\frac{2 l+1}{2} \sum_{j=1}^{n} \lambda_{j} \ell_{k}\left(x_{j}\right) P_{l}\left(x_{j}\right)=\frac{2 l+1}{2} \lambda_{k} P_{l}\left(x_{k}\right) .
$$

Hence,

$$
\begin{aligned}
\omega_{k}(x) & =\int_{-1}^{1} K(x, y) \ell_{k}(y) d y \\
& =\frac{1}{2} \int_{-1}^{1} K(x, y)\left[\sum_{l=0}^{n-1}(2 l+1) \lambda_{k} P_{l}\left(x_{k}\right) P_{l}(y)\right] d y \\
& =\frac{1}{2} \lambda_{k} \sum_{l=0}^{n-1}(2 l+1) P_{l}\left(x_{k}\right) \int_{-1}^{1} K(x, y) P_{l}(y) d y \\
& =\frac{1}{2} \lambda_{k} \sum_{l=0}^{n-1}(2 l+1) P_{l}\left(x_{k}\right) \mu_{l}(x)
\end{aligned}
$$

which completes the proof. 
Theorem 6. Let $K(x, y)$ denote an integration kernel that may have a hyper-singularity in $(-1,1)$ or at the endpoints, or is nearly singular at the endpoints. Then, we have the approximation

$$
\int_{-1}^{1} K(x, y)(w(y)-w(x)) d y \approx \sum_{k=1}^{n} \bar{\omega}_{k}(x) w\left(x_{k}\right)
$$

where $\left\{x_{k}\right\}_{k=1}^{n}$ denote the zeros of Legendre polynomials $P_{n}(x)$ of degree $n$, and

$$
\bar{\omega}_{k}(x)=\frac{1}{2} \lambda_{k} \sum_{l=0}^{n-1}(2 l+1) P_{l}\left(x_{k}\right) \bar{\mu}_{l}(x)
$$

with $\lambda_{k}$ denoting the classical Christoffel numbers associated with the $n$-point Gauss-Legendre formula and

$$
\bar{\mu}_{l}(x)=\int_{-1}^{1} K(x, y)\left(P_{l}(y)-P_{l}(x)\right) d y=\mu_{l}(x)-P_{l}(x) \mu_{0}(x) .
$$

Proof. Let $\Pi_{k} w(x)$ be defined as in the proof of Theorem 5; then

$$
\int_{-1}^{1} K(x, y)(w(y)-w(x)) d y \approx \int_{-1}^{1} K(x, y)\left(\Pi_{k} w(y)-\Pi_{k} w(x)\right) d y=\sum_{k=1}^{n} w\left(x_{k}\right) \bar{\omega}_{k}(x),
$$

where $\bar{\omega}_{k}(x)=\int_{-1}^{1} K(x, y)\left(\ell_{k}(y)-\ell_{k}(x)\right) d y$. By using (15) and (16), we can easily obtain (18) and (19).

Remark 7. We refer to (14) and (17) as n-point product quadrature rules; they both have algebraic precision at least $n-1$, i.e., they are exact for all polynomials of degree less than or equal to $n-1$.

The key to making use of the product rules (14) and (17) is to obtain explicit expressions for $\mu_{l}(x)$ and $\bar{\mu}_{l}(x)$. The Legendre polynomial $P_{l}(x)$ has the explicit form

$$
P_{l}(x)=2^{-l} \sum_{k=0}^{\lfloor l / 2\rfloor}(-1)^{k}\left(\begin{array}{l}
l \\
k
\end{array}\right)\left(\begin{array}{c}
2 l-2 k \\
l
\end{array}\right) x^{l-2 k} .
$$

Then, after some straightforward calculations, we obtain

$$
P_{l}(y)-P_{l}(x)=2^{-l} \sum_{k=0}^{\lfloor l / 2\rfloor}(-1)^{k}\left(\begin{array}{l}
l \\
k
\end{array}\right)\left(\begin{array}{c}
2 l-2 k \\
l
\end{array}\right) \sum_{m=1}^{l-2 k}\left(\begin{array}{c}
l-2 k \\
m
\end{array}\right) x^{l-2 k-m}(y-x)^{m},
$$

From (19) and (20), we obtain

$$
\begin{gathered}
\bar{\mu}_{l}(x)=2^{-l} \sum_{k=0}^{\lfloor l / 2\rfloor}(-1)^{k}\left(\begin{array}{l}
l \\
k
\end{array}\right)\left(\begin{array}{c}
2 l-2 k \\
l
\end{array}\right) \sum_{m=1}^{l-2 k}\left(\begin{array}{c}
l-2 k \\
m
\end{array}\right) x^{l-2 k-m} \theta_{m}(x) \\
\theta_{m}(x)=\int_{-1}^{1} K(x, y)(y-x)^{m} d y .
\end{gathered}
$$

There remains the need to obtain an explicit expression for $\theta_{m}(x)$ for which we consider three cases. 
Case 1: If $K(x, y)=|x-y|^{-1-2 s}$ and $x, y \in(-1,1)$, we have

$$
\begin{gathered}
\theta_{0}(x)= \begin{cases}\ln \left(1-x^{2}\right) & \text { for } s=0 \\
\frac{1}{-2 s}\left((1-x)^{-2 s}+(1+x)^{-2 s}\right) & \text { for } s \in(0,1),\end{cases} \\
\theta_{1}(x)= \begin{cases}\ln \left|\frac{1-x}{1+x}\right| & \text { for } s=\frac{1}{2} \\
\frac{1}{1-2 s}\left((1-x)^{1-2 s}-(1+x)^{1-2 s}\right) & \text { for } s \in[0,1) \backslash \frac{1}{2}\end{cases}
\end{gathered}
$$

and, for $m \geq 2$,

$$
\theta_{m}(x)=\frac{1}{m-2 s}\left((1-x)^{m-2 s}+(-1)^{m}(1+x)^{m-2 s}\right) .
$$

Case 2: If $K(x, y)=(y-x)^{-1-2 s}, y \in(-1,1)$, and $x<-1$, we have

$$
\begin{gathered}
\theta_{0}(x)= \begin{cases}\ln \left|\frac{1-x}{1+x}\right| & \text { for } s=0 \\
\frac{1}{-2 s}\left((1-x)^{-2 s}-(-1-x)^{-2 s}\right) & \text { for } s \in(0,1),\end{cases} \\
\theta_{1}(x)= \begin{cases}2 & \text { for } s=0 \\
\ln \left|\frac{1-x}{1+x}\right| & \text { for } s=\frac{1}{2} \\
\frac{1}{1-2 s}\left((1-x)^{1-2 s}-(-1-x)^{1-2 s}\right) & \text { for } s \in(0,1) \backslash \frac{1}{2}\end{cases}
\end{gathered}
$$

and, for $m \geq 2$,

$$
\theta_{m}(x)=\frac{1}{m-2 s}\left((1-x)^{m-2 s}-(-1-x)^{m-2 s}\right) .
$$

Case 3: If $K(-1, y)=(y+1)^{-2 s}$ and $y \in(-1,1)$, we have

$$
\theta_{0}(x)= \begin{cases}\ln 2 & \text { for } s=\frac{1}{2} \\ \frac{2^{1-2 s}}{1-2 s} & \text { for } s \in[0,1) \backslash \frac{1}{2}\end{cases}
$$

and, for $m \geq 1$,

$$
\theta_{m}(x)=\frac{2^{m+1-2 s}}{m+1-2 s}
$$

which leads to

$$
\mu_{l}^{(s)}(-1)= \begin{cases}c_{l}+(-1)^{l} \ln 2 & \text { for } s=\frac{1}{2} \\ \frac{2^{1-2 s}}{1-2 s} \prod_{k=0}^{i-1} \frac{2 s+k}{2 s-k-2} & \text { for } s \in[0,1) \backslash \frac{1}{2}\end{cases}
$$

with

$$
c_{l}=2^{-l} \sum_{k=0}^{\lfloor l / 2\rfloor}(-1)^{k}\left(\begin{array}{l}
l \\
k
\end{array}\right)\left(\begin{array}{c}
2 l-2 k \\
l
\end{array}\right) \sum_{i=1}^{l-2 k}(-1)^{l-2 k-i}\left(\begin{array}{c}
l-2 k \\
i
\end{array}\right) \frac{2^{i}}{i} .
$$

The first few values of $c_{l}$ are given by $c_{0}=0, c_{1}=2, c_{2}=-3, c_{3}=\frac{11}{3}$, and $c_{4}=-\frac{25}{6}$. Moreover, for $K(1, y)=(1-y)^{-2 s}$ and $y \in(-1,1)$, we have

$$
\mu_{l}^{(s)}(1)=(-1)^{l} \mu_{l}^{(s)}(-1) .
$$




\subsection{Double exponential rule}

The double exponential (DE) rule was first introduced in [38] for approximating integrals with weak singularities at the endpoints. Consider the integral $I=\int_{-1}^{1} u(x) d x$ where $u(x)$ is analytic on $(-1,1)$ but may have a weakly singularity at the endpoint $x=-1$ or $x=1$ or both. Applying the transformation of variables

$$
x=\psi(t), \quad \lim _{t \rightarrow-\infty} \psi(t)=-1, \quad \text { and } \quad \lim _{t \rightarrow \infty} \psi(t)=1,
$$

where $\psi(t)$ is analytic on $(-\infty, \infty)$, we then have

$$
I=\int_{-\infty}^{\infty} u(\psi(t)) \psi^{\prime}(t) d t
$$

We choose the transformation $x=\psi(t)=\tanh (\pi / 2 \sinh t)$ which results in a double exponential decay of the integrand. Note that

$$
\psi^{\prime}(t)=\frac{\pi / 2 \cosh t}{\cosh ^{2}(\pi / 2 \sinh t)} .
$$

Applying the trapezoidal formula with a uniform mesh size $h$ to (24) yields

$$
I_{h}=h \sum_{k=-\infty}^{\infty} u(\psi(k h)) \psi^{\prime}(k h) .
$$

We often truncate the infinite summation by choosing an integer $N_{d e}>0$ and set

$$
I_{h}^{\left(N_{d e}\right)}=h \sum_{k=-N_{d e}}^{N_{d e}} u(\psi(k h)) \psi^{\prime}(k h) .
$$

The reason this rule is referred to as a double-exponential rule is that the integrand, after the transformation of variables, decays double exponentially, i.e.,

$$
\left|u(\psi(t)) \psi^{\prime}(t)\right| \leq e^{-c e^{|t|}} \quad \text { as }|t| \rightarrow \infty .
$$

\section{Galerkin finite element discretizations}

Let $S\left(\Omega^{\prime}\right)$ denote the "energy" or "solution" space

$$
S\left(\Omega^{\prime}\right)=\left\{v \in L^{2}\left(\Omega^{\prime}\right):\|v\| \|<\infty\right\}
$$

where

$$
\|\mid v\|^{2}=\frac{1-s}{\delta^{2-2 s}} \int_{\Omega} \int_{\Omega^{\prime} \cap B_{\delta}(x)} \frac{(v(y)-v(x))^{2}}{|x-y|^{1+2 s}} d y d x .
$$

Define the affine space $S_{g}\left(\Omega^{\prime}\right)=\left\{u(x) \in S\left(\Omega^{\prime}\right): u(x)=g(x)\right.$ a.e. on $\left.\Omega_{\mathcal{I}}\right\}$ and the subspace $S_{0}=\left\{v(x) \in S\left(\Omega^{\prime}\right): v(x)=0\right.$ in $\left.\Omega_{\mathcal{I}}\right\}$. Then, the Galerkin variational problem corresponding to (2) is defined as:

$$
\begin{aligned}
& \text { given } f(x) \in L^{2}(\Omega) \text { and } g(x) \in L^{2}\left(\Omega_{\mathcal{I}}\right) \text {, find } u(x) \in S_{g}\left(\Omega^{\prime}\right) \text { satisfying } \\
& -\frac{2-2 s}{\delta^{2-2 s}} \int_{\Omega} v(x) \int_{\Omega^{\prime} \cap B_{\delta}(x)} \frac{u(y)-u(x)}{|x-y|^{1+2 s}} d y d x=\int_{\Omega} f(x) v(x) d x, \quad \forall v \in S_{0} .
\end{aligned}
$$


For $s \in(0,1)$, it is known (see, e.g., [9]) that $S\left(\Omega^{\prime}\right)=H^{s}\left(\Omega^{\prime}\right)$, the fractional Sobolev space of order $s$. For $s=0$, no known characterization of the solution space in terms of standard function spaces is known; however, it is known that $S\left(\Omega^{\prime}\right)$ is a Hilbert space and that it is a strict subspace of $L^{2}\left(\Omega^{\prime}\right)$.

Let $S^{h} \subset S\left(\Omega^{\prime}\right)$ denote a family of finite-dimensional subspaces parameterized by $h \rightarrow 0$. For $x \in \Omega_{\mathcal{I}}$, let $g^{h}(x)$ denote an approximation of $g(x)$; if $g(x)$ is sufficiently smooth, we can choose $\left.g^{h}(x) \in S^{h}\right|_{\Omega_{\mathcal{I}}}$ to be an interpolant of $g(x)$; otherwise, we can choose $\left.g^{h}(x) \in S^{h}\right|_{\Omega_{\mathcal{I}}}$ to be the $L^{2}\left(\Omega_{\mathcal{I}}\right)$ projection of $g(x)$. We then define the affine space $S_{g}^{h}=\left\{u^{h}(x) \in S^{h}: u^{h}(x)=\right.$ $g^{h}(x)$ a.e. on $\left.\Omega_{\mathcal{I}}\right\}$ and the subspace $S_{0}^{h}=\left\{v^{h}(x) \in S^{h} \mid v^{h}(x)=0\right.$ a.e. on $\left.\Omega_{\mathcal{I}}\right\} \in S_{0}\left(\Omega^{\prime}\right)$. Then, the Galerkin discretization of (26) is given by:

$$
\begin{aligned}
& \text { Given } f(x) \in L^{2}(\Omega) \text { and } g(x) \in L^{2}\left(\Omega_{\mathcal{I}}\right) \text {, find } u^{h}(x) \in S_{g}^{h} \text { satisfying } \\
& -\frac{2-2 s}{\delta^{2-2 s}} \int_{\Omega} v^{h}(x) \int_{\Omega^{\prime} \cap B_{\delta}(x)} \frac{u^{h}(y)-u^{h}(x)}{|x-y|^{1+2 s}} d y d x=\int_{\Omega} f(x) v^{h}(x) d x, \quad \forall v^{h}(x) \in S_{0}^{h} .
\end{aligned}
$$

Define a partition of $\Omega^{\prime}$ such that

$$
-\delta=x_{-K}<\cdots<x_{-1}<0=x_{0}<x_{1}<\cdots<x_{N}<x_{N+1}=1<x_{N+2}<\cdots<x_{N+K+1}=1+\delta
$$

and let $h_{i}=x_{i}-x_{i-1}$ denote the mesh size of the element $e_{i}=\left(x_{i-1}, x_{i}\right)$ and let $h$ denote the maximum length of any of elements $e_{i}, i=-K+1, \ldots, N+K+1$.

\subsection{Continuous piecewise-linear finite element method}

Choose $S^{h}$ to be the space of continuous piecewise-linear polynomials defined with respect to the partition (28) and choose the standard "hat" functions as a basis which we denote by $\left\{\phi_{j}(x)\right\}_{j=-K+1}^{N+K+1}$. Let $u^{h}(x)=\sum_{j=-K+1}^{N+K+1} u_{j} \phi_{j}(x)$ and set $u_{j}=g^{h}\left(x_{j}\right)$ for $x_{j} \in \bar{\Omega}_{\mathcal{I}}$, i.e., for $j=$ $-K, \ldots, 0$ and $j=N+1, \ldots, N+K+1$. Furthermore, for $i=1, \ldots, N$, let $v^{h}(x)=\phi_{i}(x)$. Then, (27) is equivalent to

$$
\begin{gathered}
\frac{2-2 s}{\delta^{2-2 s}} \sum_{j=-K+1}^{N+K+1} u_{j}\left(\int_{e_{i}^{*}} \phi_{i}(x) \phi_{j}(x) d x \int_{B(x ; \delta) \backslash e_{j}^{*}} \frac{1}{|y-x|^{1+2 s}} d y\right. \\
\left.-\int_{e_{i}^{*}} \phi_{i}(x) \int_{B(x ; \delta) \cap e_{j}^{*}} \frac{\phi_{j}(y)-\phi_{j}(x)}{|y-x|^{1+2 s}} d y d x\right) \\
=\int_{e_{i}^{*}} \phi_{i}(x) f(x) d x, \quad \text { for } i=1,2, \ldots, N,
\end{gathered}
$$

where $e_{i}^{*}=e_{i} \cup e_{i+1}$.

Let $\vec{U}$ denote the $N$-vector having as components the unknown coefficients $u_{j}$ for $j=1, \ldots, N$. Let the entries of the $N \times N$ stiffness matrix $\mathbb{A}$ defined by

$$
\begin{aligned}
a_{i j}=\frac{2-2 s}{\delta^{2-2 s}} & \left(\int_{e_{i}^{*}} \phi_{i}(x) \phi_{j}(x) \int_{B(x ; \delta) \backslash e_{j}^{*}} \frac{1}{|y-x|^{1+2 s}} d y d x\right. \\
& \left.-\int_{e_{i}^{*}} \phi_{i}(x) \int_{B(x ; \delta) \cap e_{j}^{*}} \frac{\phi_{j}(y)-\phi_{j}(x)}{|y-x|^{1+2 s}} d y d x\right)
\end{aligned}
$$


for $i, j=1, \ldots, N$ and let the entries of the right-hand side $N$-vector $\vec{F}$ be defined by

$$
\begin{aligned}
f_{i}= & \int_{e_{i}^{*}} \phi_{i}(x) f(x) d x \\
& +\frac{2-2 s}{\delta^{2-2 s}}\left(\sum_{j=-K+1}^{0}+\sum_{j=N+2}^{N+K+1}\right) g^{h}\left(x_{j}\right) \int_{e_{i}^{*}} \phi_{i}(x) \int_{B(x ; \delta) \cap e_{j}^{*}} \frac{\phi_{j}(y)}{|y-x|^{1+2 s}} d y d x
\end{aligned}
$$

for $i=1,2, \ldots, N$. Then, (29) can be expressed in the form

$$
\mathbb{A} \vec{U}=\vec{F}
$$

\subsection{Discontinuous piecewise-linear finite element method}

We again use the partition of $\Omega^{\prime}$ defined in (28). A basis for the space of discontinuous piecewiselinear functions is given by, $j=-K+1, \ldots, N+K+1$,

$$
\phi_{2 j-2}(x)= \begin{cases}\frac{x_{j}-x}{x_{j}-x_{j-1}} & \text { for } x \in e_{j} \\ 0 & \text { otherwise }\end{cases}
$$

and

$$
\phi_{2 j-1}(x)= \begin{cases}\frac{x-x_{j-1}}{x_{j}-x_{j-1}} & \text { for } x \in e_{j} \\ 0 & \text { otherwise. }\end{cases}
$$

Note that the support of the $j$-th basis function is the single element $e_{j}$. Now $\mathbb{A}$ is an $(2 N+2) \times$ $(2 N+2)$ matrix and $\vec{U}$ and $\vec{b}$ are $(2 N+2)$-vectors. For the sake of brevity, we do not write down formulas for the entries of the stiffness matrix $\mathbb{A}$.

Remark 8. We note the following.

1. It is important to observe that the space of discontinuous piecewise-linear functions contains the space of continuous piecewise-linear functions.

2. Also important to observe is that, for $s<\frac{1}{2}$, the space of discontinuous finite element functions is a subspace of the solution space $S\left(\Omega^{\prime}\right)$, i.e., that space is conforming for the problem (26). This is in contrast to elliptic PDEs for which that space is not conforming, i.e., it is not a subspace of the solution space $H^{1}(\Omega)$ for that case.

3. In practice, it is easy to convert a code that implements continuous piecewise-linear finite element approximations into one that implements the discontinuous piecewise-linear finite element approximations, because the calculations of the double integrals appearing in the entries of the stiffness matrix $\mathbb{A}$ are the same, the only difference being in the actual assembly of that matrix. This may be an advantage over the implementations of [12, 24] because in those implementation, the two approximations are separately treated.

4. We here investigate the special structure of the stiffness matrix $\mathbb{A}$ under the uniform mesh. Denote by

$$
T=T\left(t_{-n+1}, t_{-2 n}, \cdots, t_{0}, t_{1}, \cdots, t_{n-1}\right)
$$

an $n \times n$ Toeplitz matrix $T=\left(\alpha_{i j}\right)$ whose entries are specified by $\alpha_{i j}=t_{i-j}$. When $T$ is symmetric, (32) is often simplified as

$$
S T\left(t_{0}, t_{1}, \cdots, t_{n-1}\right) .
$$


If the continuous Galerkin method is applied, then $\mathbb{A}$ has a Toeplitz structure as

$$
\mathbb{A}=S T\left(a_{11}, a_{12}, \cdots, a_{1 N}\right)
$$

If the discontiuous Galerkin method is applied, $\mathbb{A}$ has a nearly Toeplitz structure, with the form

$$
\mathbb{A}=D_{0}+D_{1} T_{1}+T_{1}^{\prime} D_{1}+D_{2} T_{2}+T_{2}^{\prime} D_{2}
$$

where $D_{0}=a_{11} \mathbb{I}$ where $\mathbb{I}$ denotes the $(2 N+2) \times(2 N+2)$ identity matrix, $D_{1}$ and $D_{2}$ are $(2 N+2) \times(2 N+2)$ diagonal matrices and $T_{1}, T_{1}^{\prime}, T_{2}, T_{2}^{\prime}$ are $(2 N+2) \times(2 N+2)$ Toeplitz matrices, defined by

$$
\begin{aligned}
& D_{1}=\operatorname{diag}(1,0,1, \cdots, 1,0), \quad D_{2}=\operatorname{diag}(0,1,0, \cdots, 0,1), \\
& T_{1}=T(\underbrace{0, \cdots, 0}_{2 N+2}, a_{12}, a_{13}, \cdots, a_{1,2 N+1}, a_{1,2 N+2}) \\
& T_{1}^{\prime}=T(a_{2 N+2,1}, a_{2 N+1,1}, \cdots, a_{41}, a_{31}, \underbrace{0, \cdots, 0}_{2 N+2}) \\
& T_{2}=T(\underbrace{0, \cdots, 0}_{2 N+2}, a_{23},, a_{24}, \cdots, a_{2,2 N+1}, a_{2,2 N+2}, 0) \\
& T_{2}^{\prime}=T(0, a_{2 N+2,2}, a_{2 N+1,2}, \cdots, a_{42}, a_{32}, \underbrace{0, \cdots, 0}_{2 N+2}) .
\end{aligned}
$$

Because $\mathbb{A}$ has the Toeplitz structure (33) or the near Toeplitz structure (34), the multiplication of a vector by $\mathbb{A}$ can be performed by fast Fourier transformation (FFT) with the computational cost $O(N \ln N)$ in the solution of the linear system by iterative solvers.

\subsection{Theoretical convergence behavior}

Let the exact solution $u \in H^{\alpha}\left(\Omega^{\prime}\right)$ for $\alpha>0$ and $r=\min \{2, \alpha\}$. Then, from [15], we have

$$
\left\|u-u^{h}\right\|_{L^{2}\left(\Omega^{\prime}\right)} \leq C h^{r-\gamma} \delta^{-1+\gamma}\|u\|_{H^{\alpha}\left(\Omega^{\prime}\right)}
$$

for any $0<\gamma<1$, where $C$ is independent of $h$ and $\delta$. For fixed $\delta$, we see that, by choosing $0<\gamma=\epsilon \ll 1$, that the error estimate (35) implies that, with $C_{\delta}=O\left(\delta^{-1+\epsilon}\right)$,

$$
\left\|u-u^{h}\right\|_{L^{2}\left(\Omega^{\prime}\right)} \leq C_{\delta} h^{r-\epsilon}\|u\|_{H^{\alpha}\left(\Omega^{\prime}\right)} .
$$

Note that the estimate (36) is not uniform in $\delta$. For $\delta$ a constant multiple of $h$, we have, with $C$ independent of $h$ and $\delta$,

$$
\left\|u-u^{h}\right\|_{L^{2}\left(\Omega^{\prime}\right)} \leq C h^{r-1}\|u\|_{H^{\alpha}\left(\Omega^{\prime}\right)} .
$$

If $\delta$ is fixed independent of $h$, the estimate (35) is nearly optimal with respect to the convergence in $h$ because the error in the best approximation to a function $u \in H^{r}\left(\Omega^{\prime}\right)$ is of $O\left(h^{r}\right)$, i.e., (35) "misses" optimality by an arbitrarily small reduction in the exponent. On the other hand, the estimate (37) for the case of $\delta$ being proportional to $h$ is suboptimal, i.e., the exponent is one less than that for the best approximation. 


\section{Efficient computation of the stiffness matrix entries}

From (29), we see that it suffices to consider the approximation of the double integrals

$$
\mathcal{I}=\int_{e_{m}} \phi_{i}(x) \phi_{j}(x) \int_{B(x ; \delta) \backslash e_{m}} \frac{1}{|y-x|^{1+2 s}} d y d x
$$

and

$$
\mathcal{J}=\int_{e_{m}} \phi_{i}(x) \int_{e_{n} \cap B(x ; \delta)} \frac{\phi_{j}(y)-\phi_{j}(x)}{|y-x|^{1+2 s}} d y d x
$$

where $i=m-1$ or $m$ and $j=n-1$ or $n$ for continuous piecewise-linear finite element approximations, and $i=2 m-2$ or $2 m-1$ and $j=2 n-2$ or $2 n-1$ for discontinuous piecewise-linear approximations. For simplicity, in (39), we normalize the intervals of the integration to $(0,1)$ and denote the normalized basis functions $\varphi^{(k)}(\tau)$ and $\varphi^{(l)}(t)$ with the new normalized variables $\tau$ and $t$ corresponding to the original basis functions $\phi_{i}(x)$ and $\phi_{j}(y)$, respectively.

5.1. $n=m$, i.e., the two elements $e_{m}$ and $e_{n}=e_{m}$ coincide

The integral (38) consists of two parts:

$$
\begin{aligned}
\mathcal{I}=\int_{x_{m-1}}^{\min \left(x_{m}, x_{m-1}+\delta\right)} \phi_{i}(x) \phi_{j}(x) \int_{x-\delta}^{x_{m-1}} \frac{1}{(x-y)^{1+2 s}} d y d x \\
\quad+\int_{\max \left(x_{m-1}, x_{m}-\delta\right)}^{x_{m}} \phi_{i}(x) \phi_{j}(x) \int_{x_{m}}^{x+\delta} \frac{1}{(y-x)^{1+2 s}} d y d x .
\end{aligned}
$$

Taking integration for the inner integrals in (40) and then using some appropriate transformations of variables, we have

$$
\mathcal{I}= \begin{cases}h_{m} \int_{0}^{\min \left(1, \frac{\delta}{h_{m}}\right)} \Phi(\tau)\left(\ln \frac{\delta}{h_{m}}-\ln \tau\right) d \tau, & s=0 \\ -h_{m} \int_{0}^{\min \left(1, \frac{\delta}{h_{m}}\right)} \Phi(\tau)\left(\delta^{-1}-h_{m}^{-1} \tau^{-1}\right) d \tau+\Phi(0) \ln h_{m}, & s=0.5 \\ \frac{h_{m}}{-2 s} \int_{0}^{\min \left(1, \frac{\delta}{h_{m}}\right)} \Phi(\tau)\left(\delta^{-2 s}-h_{m}^{-2 s} \tau^{-2 s}\right) d \tau, & \text { otherwise }\end{cases}
$$

where $\Phi(\tau)=\varphi^{(k)}(\tau) \varphi^{(l)}(\tau)+\varphi^{(k)}(1-\tau) \varphi^{(l)}(1-\tau)$. The first part in $\mathcal{I}$ can be evaluated by a classical Gaussian quadrature rule, and the second part has the endpoint singularity, which can be evaluated by DE rule if $s=0$, and otherwise by the three-point product rule (14) corresponding to Case 3.

For (39), it can be split into three parts:

$$
\begin{aligned}
& \mathcal{J}=\int_{x_{m-1}}^{x_{m}} \phi_{i}(x) \int_{x_{m-1}}^{x_{m}} \frac{\phi_{j}(y)-\phi_{j}(x)}{|y-x|^{1+2 s}} d y d x \\
&-\int_{x_{m-1}}^{\max \left(x_{m-1}, x_{m}-\delta\right)} \phi_{i}(x) \int_{x+\delta}^{x_{m}} \frac{\phi_{j}(y)-\phi_{j}(x)}{(y-x)^{1+2 s}} d y d x \\
&-\int_{\min \left(x_{m}, x_{m-1}+\delta\right)}^{x_{m}} \phi_{i}(x) \int_{x_{m-1}}^{x-\delta} \frac{\phi_{j}(y)-\phi_{j}(x)}{(x-y)^{1+2 s}} d y d x \\
&:=\mathcal{J}_{1}-\mathcal{J}_{2}-\mathcal{J}_{3} .
\end{aligned}
$$


Let $x=x_{m-1}+\tau h_{m}$ and $y=x_{m-1}+t h_{m}$ so that $e_{m}=e_{n}=(0,1)$. With these transformations of variables, using (11)-(13), we have

$$
\begin{aligned}
& \mathcal{J}_{1}=h_{m}^{1-2 s} \int_{0}^{1} \varphi^{(k)}(\tau) \int_{0}^{1} \frac{\varphi^{(l)}(t)-\varphi^{(l)}(\tau)}{|t-\tau|^{1+2 s}} d t d \tau, \\
& \mathcal{J}_{2}=h_{m}^{1-2 s} \int_{0}^{\max \left(0,1-\frac{\delta}{h_{m}}\right)} \varphi^{(k)}(\tau) \int_{\tau+\frac{\delta}{h_{m}}}^{1} \frac{\varphi^{(l)}(t)-\varphi^{(l)}(\tau)}{(t-\tau)^{1+2 s}} d t d \tau, \\
& \mathcal{J}_{3}=h_{m}^{1-2 s} \int_{0}^{\max \left(0,1-\frac{\delta}{h_{m}}\right)} \varphi^{(k)}(1-\tau) \int_{\tau+\frac{\delta}{h_{m}}}^{1} \frac{\varphi^{(l)}(1-t)-\varphi^{(l)}(1-\tau)}{(t-\tau)^{1+2 s}} d t d \tau .
\end{aligned}
$$

Clearly, $\mathcal{J}_{2}$ and $\mathcal{J}_{3}$ vanish if $\delta>h_{m}$. The inner integrals of $\mathcal{J}_{i}, i=1,2,3$, can be evaluated exactly by the two-point product rule (17) with the appropriate kernels and then we can use the DE-rule (25) to evaluate the outer one.

5.2. $n=m \pm 1$, i.e., the two elements $e_{m}$ and $e_{n}=e_{m \pm 1}$ abut

In this case, (39) can be split into two parts:

$$
\begin{aligned}
\mathcal{J}= & \int_{x_{m-1}}^{\min \left(x_{m}, x_{m-1}+\delta\right)} \phi_{i}(x) \int_{\max \left(x_{m-2}, x-\delta\right)}^{x_{m-1}} \frac{\phi_{j}(y)-\phi_{j}^{*}(x)}{(x-y)^{1+2 s}} d y d x \\
& +\int_{x_{m-1}}^{\min \left(x_{m}, x_{m-1}+\delta\right)} \phi_{i}(x) \phi_{j}^{*}(x) \int_{\max \left(x_{m-2}, x-\delta\right)}^{x_{m-1}} \frac{1}{(x-y)^{1+2 s}} d y d x \\
:= & \mathcal{J}_{1}+\mathcal{J}_{2}
\end{aligned}
$$

if $n=m-1$ and

$$
\begin{aligned}
\mathcal{J}= & \int_{\max \left(x_{m-1}, x_{m}-\delta\right)}^{x_{m}} \phi_{i}(x) \int_{x_{m}}^{\min \left(x_{m+1}, x+\delta\right)} \frac{\phi_{j}(y)-\phi_{j}^{*}(x)}{(y-x)^{1+2 s}} d y d x \\
& +\int_{\max \left(x_{m-1}, x_{m}-\delta\right)}^{x_{m}} \phi_{i}(x) \phi_{j}^{*}(x) \int_{x_{m}}^{\min \left(x_{m+1}, x+\delta\right)} \frac{1}{(y-x)^{1+2 s}} d y d x \\
:= & \mathcal{J}_{1}+\mathcal{J}_{2}
\end{aligned}
$$

if $n=m+1$, where $\phi_{j}^{*}(x)$ denotes the extension of $\phi_{j}(x)$ outside of $\left[x_{m}, x_{m+1}\right]$. Let $x=x_{m-1}+$ $\tau h_{m}, y=x_{n}-t h_{n}$ for $n=m-1$ and $x=x_{m}-\tau h_{m}, y=x_{n-1}+t h_{n}$ for $n=m+1$; with an appropriate transformation of variables, we then have

$$
\mathcal{J}_{1}=h_{m} h_{n}^{-2 s} \int_{0}^{\min \left(1, \frac{\delta}{h_{m}}\right)} \varphi^{(k)}(\tau) \int_{0}^{\min \left(1,-d_{m n} \tau+\frac{\delta}{h_{n}}\right)} \frac{\varphi^{(l)}(t)-\varphi_{*}^{(l)}\left(-d_{m n} \tau\right)}{\left(t+d_{m n} \tau\right)^{1+2 s}} d t d \tau
$$

and

$$
\mathcal{J}_{2}= \begin{cases}h_{m} \int_{0}^{\min \left(1, \frac{\delta}{h_{m}}\right)} \Phi(\tau)[\ln c(\tau)-\ln (\tau)] d \tau & \text { for } s=0 \\ -\int_{0}^{\min \left(1, \frac{\delta}{h_{m}}\right)} \Phi(\tau)\left[c(\tau)^{-1}-\tau^{-1}\right] d \tau+\Phi(0) \ln h_{m} & \text { for } s=0.5 \\ \frac{h_{m}^{1-2 s}}{-2 s} \int_{0}^{\min \left(1, \frac{\delta}{h_{m}}\right)} \Phi(\tau)\left[c(\tau)^{-2 s}-\tau^{-2 s}\right] d \tau & \text { otherwise }\end{cases}
$$


where $d_{m n}=\frac{h_{m}}{h_{n}}, \Phi(\tau)=\varphi^{(k)}(\tau) \varphi_{*}^{(l)}\left(-d_{m n} \tau\right), c(\tau)=\min \left(\tau+\epsilon_{1}, \frac{\delta}{h_{m}}\right)$ with $\epsilon_{1}=d_{m n}^{-1}$ and $\varphi_{*}^{(l)}$ denotes the extension of $\varphi^{(l)}$ outside $(0,1)$. $\mathcal{J}_{1}$ can be evaluated by the two-point product rule (17) corresponding to Case 2 for the inner integral and the DE-rule (25) for the outer one. The second part of $\mathcal{J}_{2}$ can be evaluated by the DE rule (25) if $s=0$ and otherwise by the three-pint product rule (14) corresponding to Case 3 . If the mesh is quasi-uniform, then $\epsilon_{1}$ is not very small, and thus the first part of $\mathcal{J}_{2}$ can be evaluated using a classical Gaussian rule.

Remark 9. In Section 6.4, we will consider a local grid refinement approach to recover the full accuracy of the discontinuous Galerkin finite element approximations, where $\epsilon_{1}>0$ may tend to zero. In this case, the first part of $\mathcal{J}_{2}$ may be nearly singular, which can be evaluated by the DE rule (25).

\section{3. $e_{m}$ and $e_{n}$ are neither coincident nor abut}

Let $x=x_{m-1}+h_{m} \tau, y=x_{n}-h_{n} t$ and $\epsilon_{2}=\frac{x_{m-1}-x_{n}}{h_{n}}$ for $n<m-1$ and let $x=x_{m}-h_{m} \tau, y=$ $x_{n-1}+h_{n} t$ and $\epsilon_{2}=\frac{x_{n-1}-x_{m}}{h_{n}}$ for $n>m+1$, then

$$
\mathcal{J}=h_{m} h_{n}^{-2 s} \int_{0}^{\min \left(1, \frac{\delta}{h_{m}}-\frac{\epsilon_{2}}{d_{m n}}\right)} \varphi^{(k)}(\tau) \int_{0}^{\min \left(1,-d_{m n} \tau-\epsilon_{2}+\frac{\delta}{h_{n}}\right)} \frac{\varphi^{(l)}(\tau)}{\left(t+d_{m n} \tau+\epsilon_{2}\right)^{1+2 s}} d t d \tau .
$$

If the grid is quasi-uniform, then $\epsilon_{2}$ is not very small, and thus $\mathcal{J}$ has no singularity and can be evaluated using classical Gaussian quadrature rules.

Remark 10. Also in Section 6.4, $\epsilon_{2}$ may be very small, which yields that the inner integral in $\mathcal{J}$ may be nearly singular. In such case, we can take the splitting trick used in Section 5.2 to compute $\mathcal{J}$.

Remark 11. In practice, very few quadrature points are needed to calculate the entries of the stiffness matrix. For example, when evaluating $\mathcal{J}_{k}, k=1,2,3$, in Section 5.1 and $\mathcal{J}_{1}$ in Section 5.2, the inner integrals can be evaluated using two quadrature points and then the outer ones are calculated by the DE-rule with $2 N_{d e}+1$ quadrature points, resulting in $4 N_{d e}+2$ total quadrature points. In practice, we can choose $N_{d e}=10 \sim 40$, depending on the singularity index $s$. Let $N_{g s}$ be the number of quadrature points for a one-dimensional classical Gaussian rule; then, the total number of quadrature points for evaluating the double integrals in Section 5.3 is $N_{g s}^{2}$. We can choose $N_{g s}=8$ in practice. Compared, e.g., with the thousands of points used in [12], there is no doubt that our method is very efficient.

\section{Numerical experiments}

We provide two numerical examples of FEMs for the model problem (2). The first manufactured solution is given by the smooth function

$$
u(x)=x^{2}\left(1-x^{2}\right)
$$

which leads to the right-hand side

$$
f_{\delta}(x)=\left(12 x^{2}-2\right)+\frac{2 s-2}{s-2} \delta^{2}
$$


for any $s \in[0,1)$. The second has a jump discontinuity:

$$
u(x)= \begin{cases}x & \text { for } x<0.5 \\ x^{2} & \text { for } x \geq 0.5\end{cases}
$$

for which, if $s \in(0,1) \backslash\{0.5\}$,

$$
f_{\delta}(x)=\left\{\begin{array}{cl}
0 & x<0.5-\delta \\
-\frac{-2 s+2}{\delta^{-2 s+2}}\left[\frac{\delta^{-2 s+2}-(0.5-x)^{-2 s+2}}{-2 s+2}+(2 x-1) \frac{\delta^{-2 s+1}-(0.5-x)^{-2 s+1}}{-2 s+1}\right. & \\
\left.+\left(x^{2}-x\right) \frac{\delta^{-2 s}-(0.5-x)^{-2 s}}{-2 s}\right] & 0.5-\delta<x<0.5 \\
-\frac{-2 s+2}{\delta^{-2 s+2}}\left[\frac{(x-0.5)^{-2 s+2}+\delta^{-2 s+2}}{-2 s+2}-(2 x-1) \frac{(x-0.5)^{-2 s+1}-\delta^{-2 s+1}}{1-2 s}\right. & \\
\left.+\left(x^{2}-x\right) \frac{(x-0.5)^{-2 s}-\delta^{-2 s}}{-2 s}\right] & 0.5<x<0.5+\delta \\
-2 & x>0.5+\delta,
\end{array}\right.
$$

and if $s=0$

$$
f_{\delta}(x)= \begin{cases}0 & x<0.5-\delta \\ -\frac{2}{\delta^{2}}\left[\frac{\delta^{2}-(0.5-x)^{2}}{2}+(2 x-1)(\delta+x-0.5)+\left(x^{2}-x\right) \ln \frac{\delta}{0.5-x}\right] & 0.5-\delta<x<0.5 \\ -\frac{2}{\delta^{2}}\left[\frac{(x-0.5)^{2}+\delta^{2}}{2}-(2 x-1)(x-0.5-\delta)+\left(x^{2}-x\right) \ln \frac{x-0.5}{\delta}\right] & 0.5<x<0.5+\delta \\ -2 & x>0.5+\delta,\end{cases}
$$

and if $s=\frac{1}{2}$

$$
f_{\delta}(x)= \begin{cases}0 & x<0.5-\delta \\ -\frac{1}{\delta}\left[\delta-(0.5-x)+(2 x-1) \ln \frac{\delta}{0.5-x}-\left(x^{2}-x\right)\left(\frac{1}{\delta}-\frac{1}{0.5-x}\right)\right] & 0.5-\delta<x<0.5 \\ -\frac{1}{\delta}\left[\delta+(x-0.5)-(2 x-1) \ln \frac{x-0.5}{\delta}-\left(x^{2}-x\right)\left(\frac{1}{x-0.5}-\frac{1}{\delta}\right)\right] & 0.5<x<0.5+\delta \\ -2 & x>0.5+\delta .\end{cases}
$$

\subsection{Numerical results for the smooth solution}

In Figure 1, we provide results of numerical experiments using continuous piecewise-linear finite element approximations for the model problem having the smooth solution (42). The horizon $\delta$ is chosen proportional to the grid size $h$; four values of $s \in[0,1)$ are chosen. For Figure 2, we have the same setup as for Figure 1, except that now three values of $\delta$ are chosen and held fixed as $h$ changes. Figures 3 and 4 repeat the setups of Figures 1 and 2, respectively, except that now discontinuous piecewise-linear finite element approximations are used. From these results, we see that for all cases, continuous and discontinuous piecewise-linear finite element approximations of the problem (2) converge at the optimal rates, i.e., we have that the $L^{2}$ and $L^{\infty}$ errors are about $O\left(h^{2}\right)$ and the $H^{1}$ errors are about $O(h)$. 


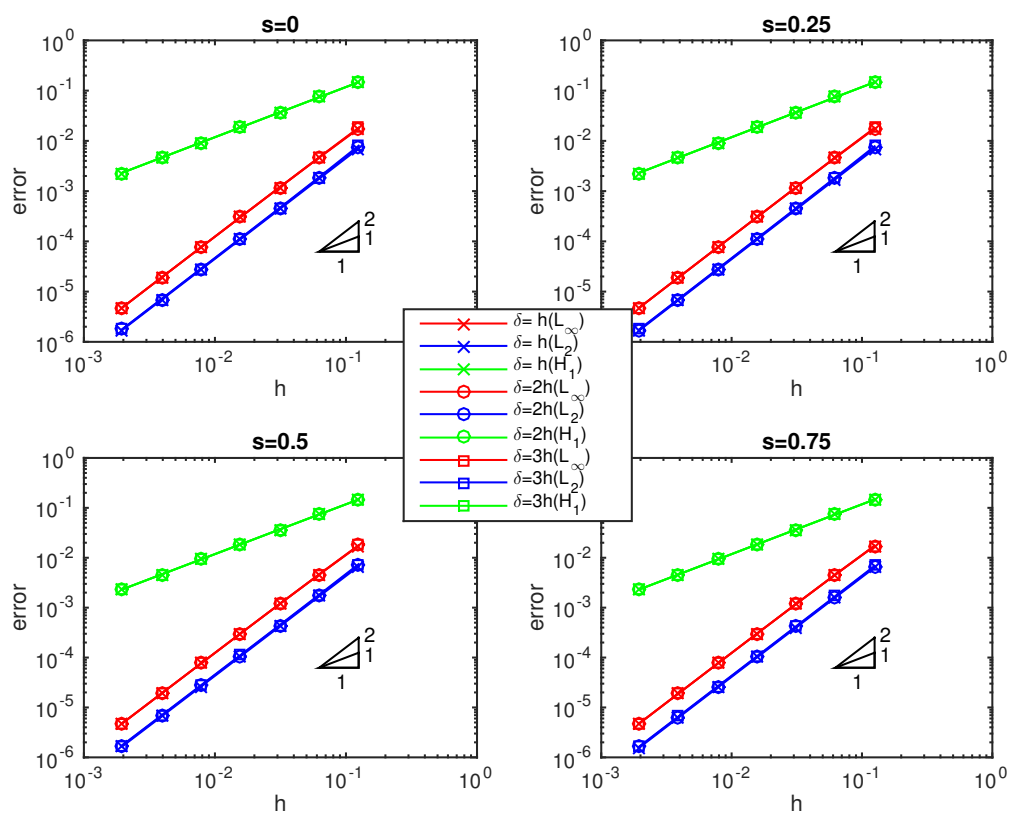

Figure 1: $L^{\infty}, L^{2}$ and $H^{1}$ errors vs. the uniform grid size $h$ for continuous piecewise-linear finite element approximations for the smooth solution (42) and for four values of $s \in[0,1)$. The horizon $\delta=h, 2 h$, or $3 h$.

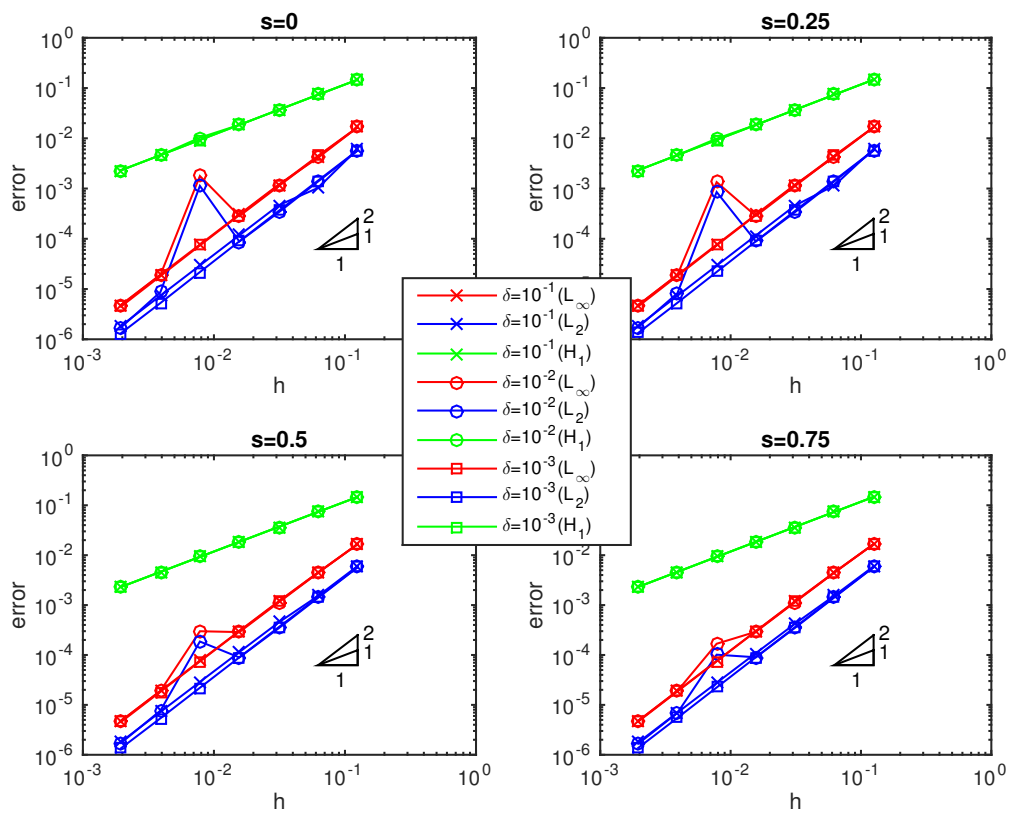

Figure 2: The same set up as for Figure 1, except that now $\delta=0.1,0.01$, or 0.001 .

\subsection{Numerical results for the discontinuous solution with grid points coinciding with the point of discontinuity}

Figures 5 to 8 have the same set ups as do Figures 1 to 4, respectively, except that now the discontinuous exact solution (43) is considered. The relation between the point at which the discontinuity in the solution occurs and the grid points is the most favorable possible, i.e., for all 


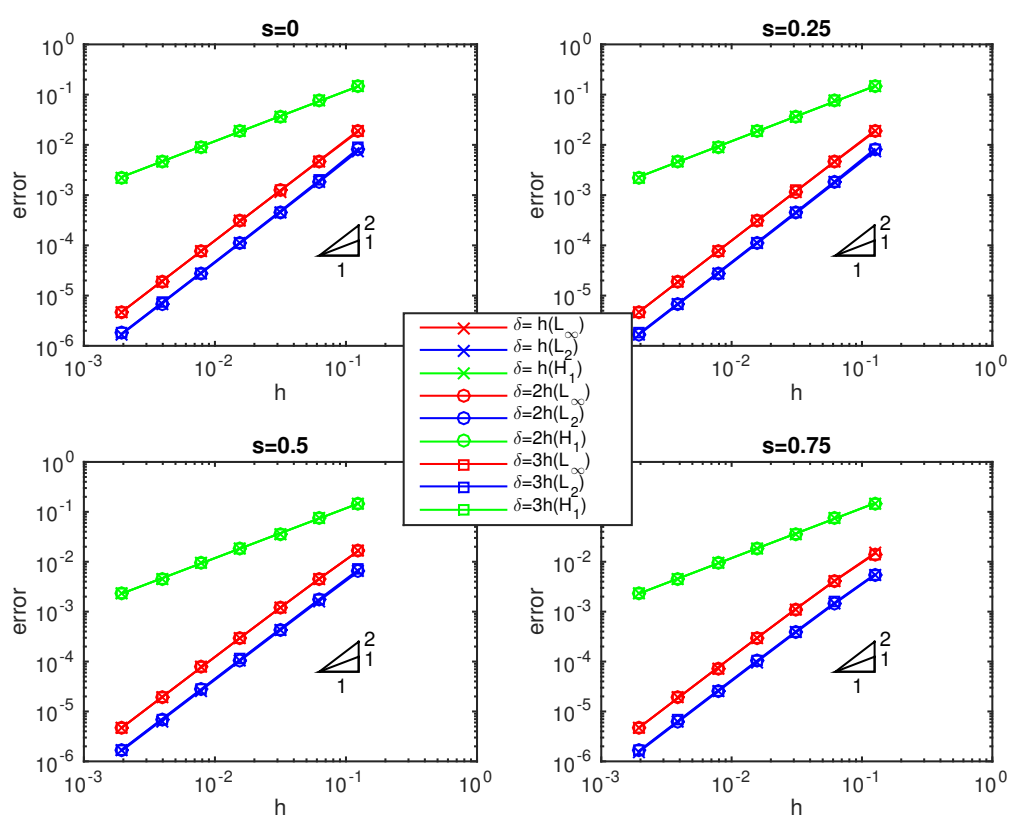

Figure 3: The same set up as for Figure 1, except that now discontinuous piecewise-linear finite element approximations are used.

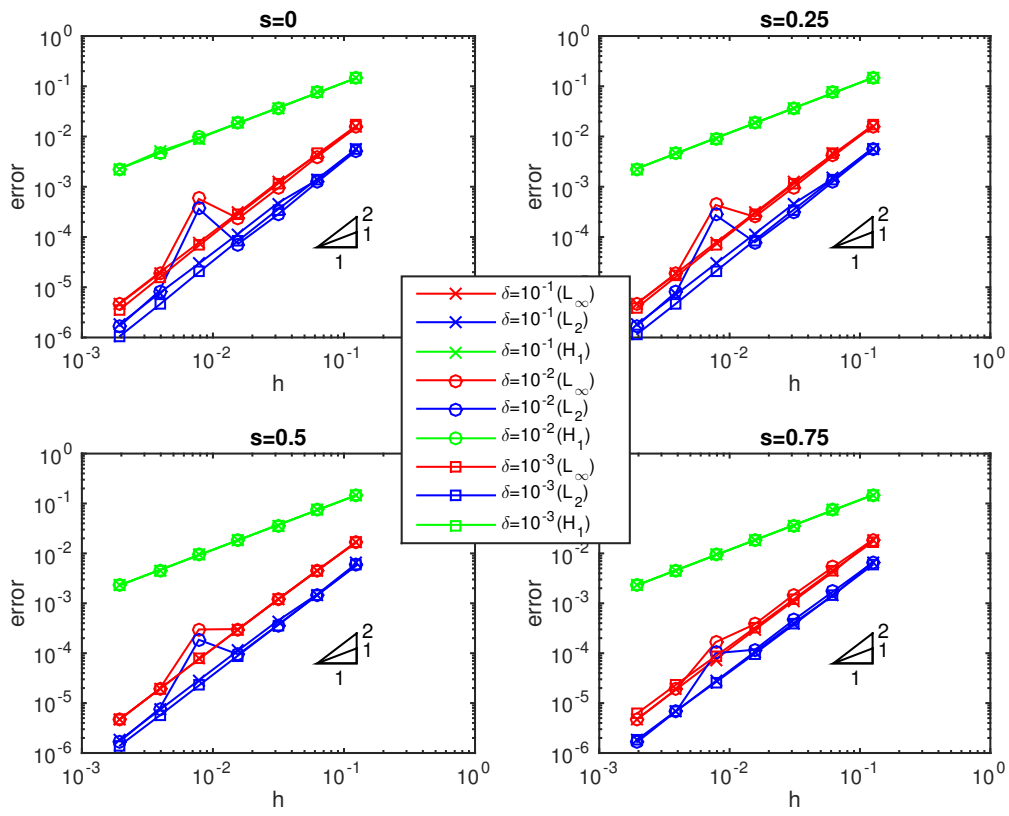

Figure 4: The same set up as for Figure 2, except that now discontinuous piecewise-linear finite element approximations are used.

grid sizes considered, the former coincides with one of the latter. Of course, in general, one would not know a priori where the discontinuity in the solution occurs, so that this best-case scenario is considered mostly to show what is the best one can expect.

From Figures 5 and 6 , we observe that in all cases, continuous piecewise linear finite element 
approximations of the linear peridynamic model (2) are seriously compromised, even though we place a grid point exactly at the point of discontinuity. The $L^{2}$ errors are about $O\left(h^{1 / 2}\right)$ and there is no convergence with respect to the $L^{\infty}$ norm. On the other hand, from Figures 7 and 8, we see that for discontinuous piecewise-linear finite element approximations, if a grid point is placed at the location of the jump discontinuity of the exact solution, one still obtains the same $L^{2}$ and $L^{\infty}$ convergence rates as those obtained for smooth solutions. These results indicate that the discontinuous piecewise-linear finite element discretization is very well suited for problems containing jump discontinuities, at least when grid points are placed at the locations of the jump discontinuities of the solution

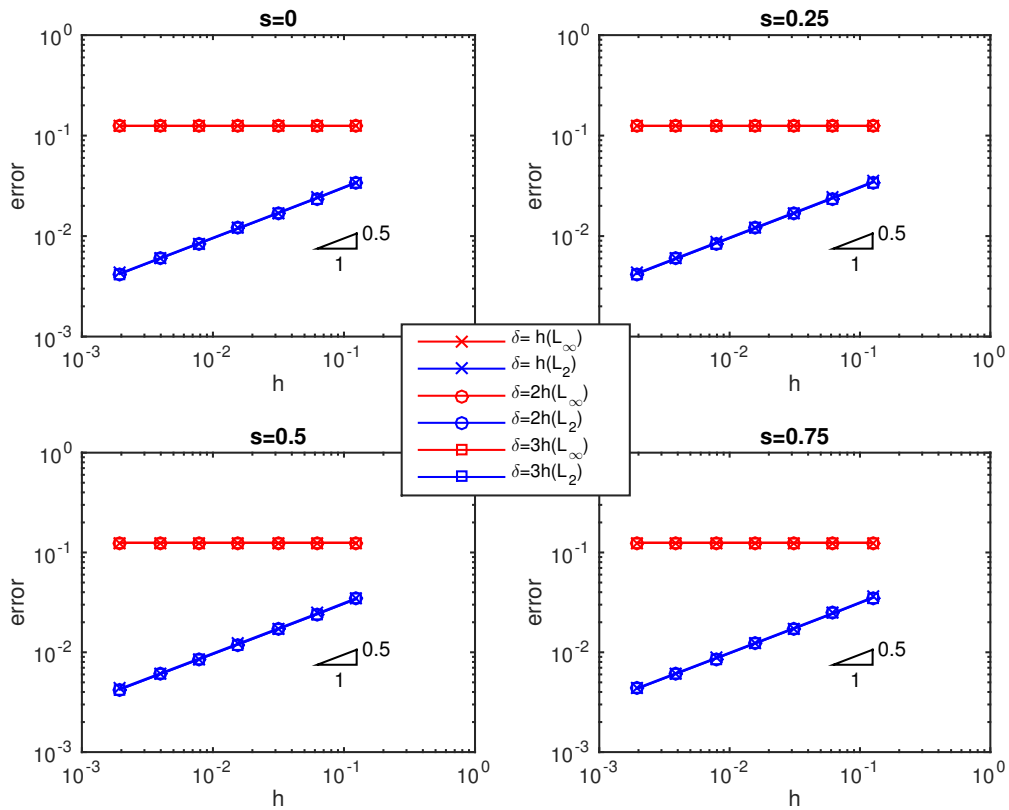

Figure 5: The same set up as for Figure 1, except that now the discontinuous exact solution (43) is considered; for all grids used, a grid point coincides with the point at which the exact solution is discontinuous.

\subsection{Numerical results for the discontinuous solution with grid points not coinciding with point of discontinuity}

In Section 6.2, we have shown that finite element discretizations of the peridynamic model has the potential of producing accurate solutions, even for discontinuous solutions. In particular, discontinuous piecewise-linear approximations are apparently robustly optimally accurate with respect to choices for the model parameter, i.e., the horizon $\delta$, the smoothness of the solution as determined by $s$, and the grid size $h$, provided that one is able to place a grid point at any point at which a jump discontinuity of the exact solution occurs. Of course, to achieve such an advantage, one has to know where the jump discontinuities occur. In one dimension, one might be able to devise some type of adaptive process to locate the points at which discontinuities occur. However, in two and higher dimensions, even if this was possible, i.e., if one could exactly locate the surfaces across which discontinuities occur, one would still not be able to recover the full accuracy possible with discontinuous piecewise-linear approximations. The reason for this is that if the surfaces of discontinuity are curved, one cannot, in general, have element faces exactly align with those surfaces. 


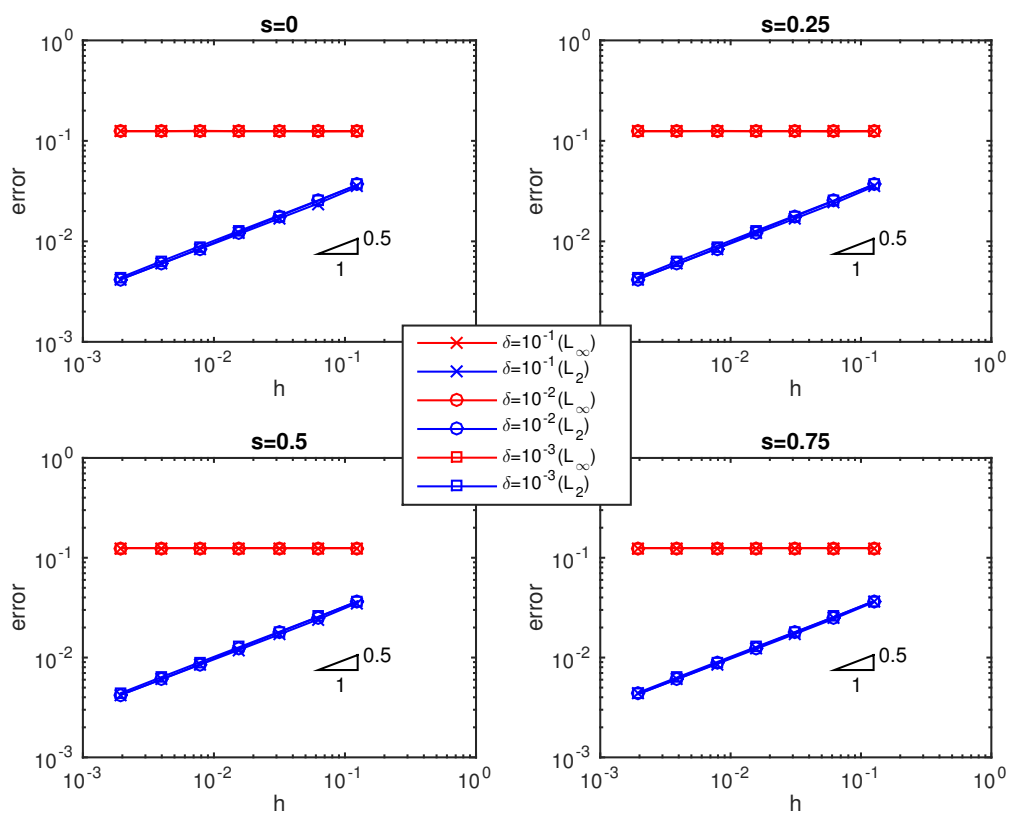

Figure 6: The same set up as for Figure 2, except that now the discontinuous exact solution (43) is considered; for all grids used, a grid point coincides with the point at which the exact solution is discontinuous.

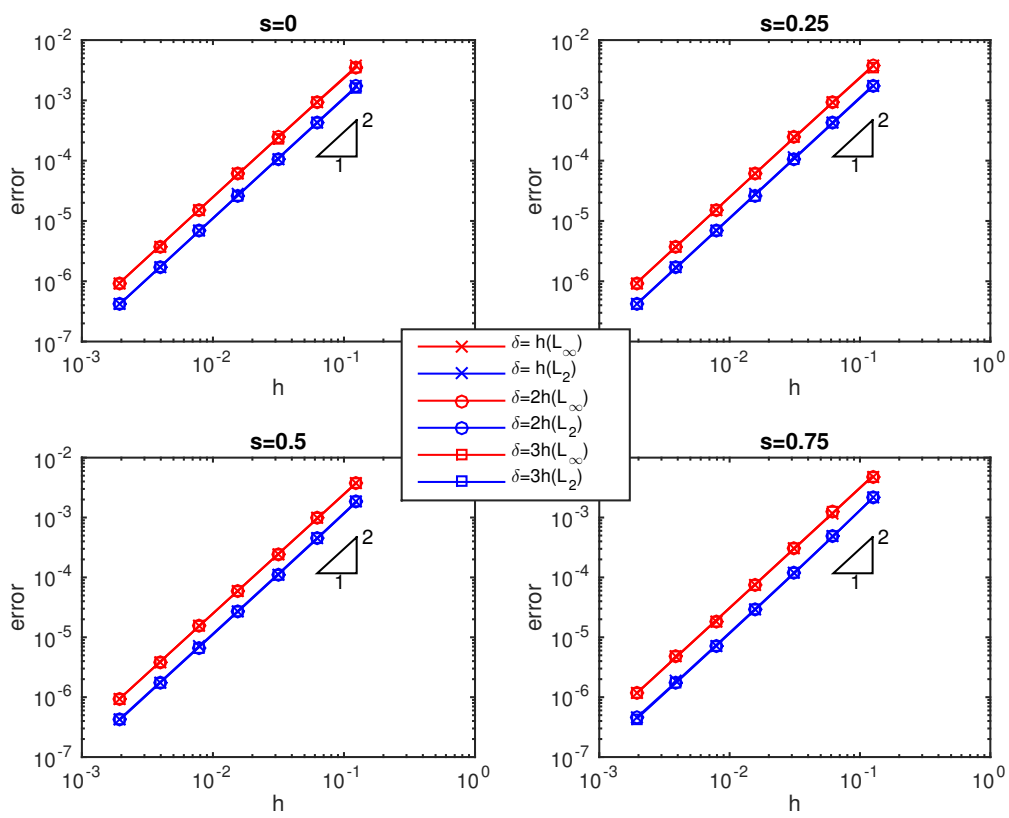

Figure 7: The same set up as for Figure 3, except that now the discontinuous exact solution (43) is considered; for all grids used, a grid point coincides with the point at which the exact solution is discontinuous.

Figures 9 to 12 provide an indication of what happen if one does not place a grid point at the location of the jump discontinuity of the solution. The set ups for these figures are exactly those for Figures 5 to 8, respectively, but now at all levels of grid refinement, no grid point coincides with the point at which the exact solution (43) is discontinuous. Now that there is no longer grid points 


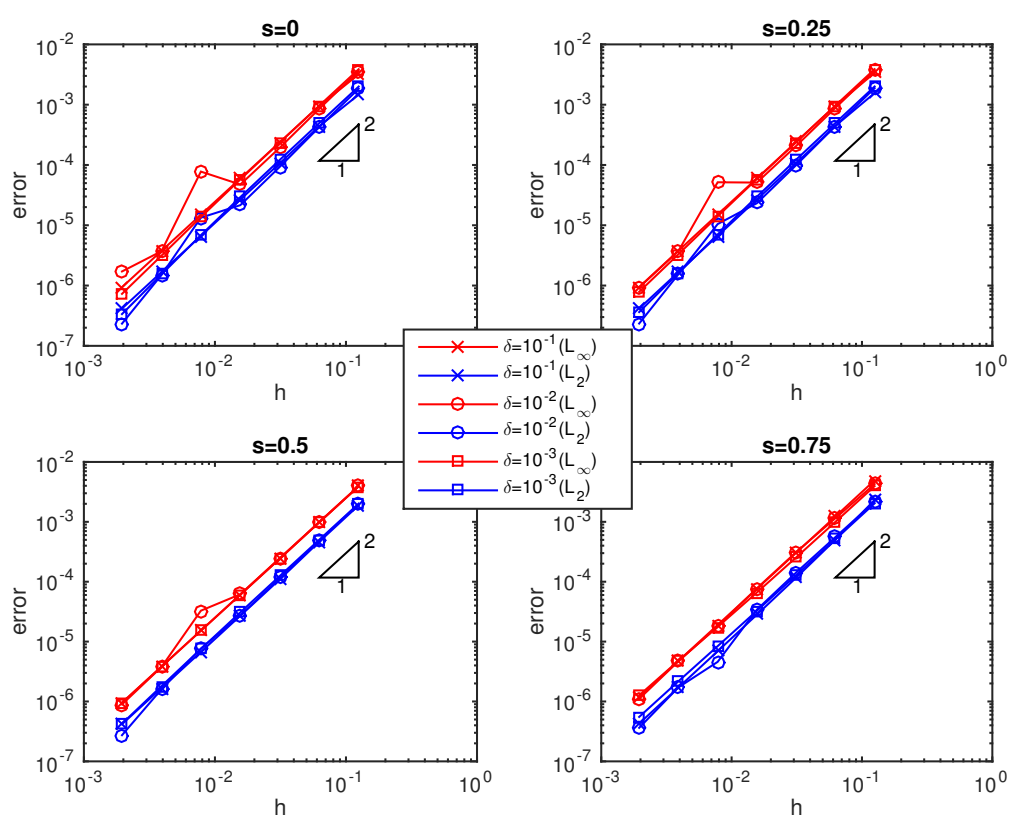

Figure 8: The same set up as for Figure 4, except that now the discontinuous exact solution (43) is considered; for all grids used, a grid point coincides with the point at which the exact solution is discontinuous.

located at discontinuity points, even discontinuous piecewise-linear discretizations suffer serious deterioration: the rate of convergence with respect to the $L^{2}$ norm deteriorates to $\frac{1}{2}$; with respect to the $L^{\infty}$ norm, no convergence is achieved. For the continuous piecewise-linear discretizations, the same results are obtained; note that these poor results were already obtained for the piecewise linear case, even if a grid point coincides with the point at which the exact solution is discontinuous; see Section 6.2.

\subsection{Full accuracy recovery}

In this section, we consider a local grid refinement approach to recover the full accuracy of the approximations. Let $\left\{E_{j}\right\},\left\{h_{j}\right\}$, and $\left\{\alpha_{j}\right\}$, denote the set of finite elements, their corresponding grid sizes, and the Sobolev smoothness index of the exact solution within each element, respectively. Define $r_{j}$ as in Section 4.3 with $\alpha$ replaced by $\alpha_{j}$. We then have that, for the best $L^{2}$ norm approximation,

$$
\inf _{v^{h} \in S^{h}}\left\|u-v^{h}\right\|_{L^{2}\left(\Omega^{\prime}\right)}^{2}=\sum_{j} \inf _{\left.v^{h} \in S^{h}\right|_{e_{j}}}\left\|u-v^{h}\right\|_{L^{2}\left(e_{j}\right)}^{2} \leq C_{\delta} \sum_{j} h_{j}^{r_{j}-\epsilon}\left\|\left.u\right|_{e_{j}}\right\|_{\alpha_{j}}^{2},
$$

where $\left.(\cdot)\right|_{e_{j}}$ denotes restriction to the element $e_{j}$. Let $e_{d}$ denote the element which contains the jump discontinuity and let $h_{d}$ denotes its length. Assume that all the other elements have length $O(h)$. In the element $e_{d}$, we have $r_{j}=1 / 2$ whereas in all the other elements we have $r_{j}=2$ for discontinuous piecewise-linear finite element discretizations. Then, (44) implies that

$$
\inf _{v^{h} \in S^{h}}\left\|u-v^{h}\right\|_{L^{2}\left(\Omega^{\prime}\right)}=O\left(h_{d}^{1 / 2-\theta}+h^{2-\theta}\right)
$$

for any $\theta>0$. Then, if we choose $h_{d}=h^{4}$, we have that

$$
\inf _{v^{h} \in S^{h}}\left\|u-v^{h}\right\|_{L^{2}\left(\Omega^{\prime}\right)}=O\left(h^{2-\theta}\right),
$$




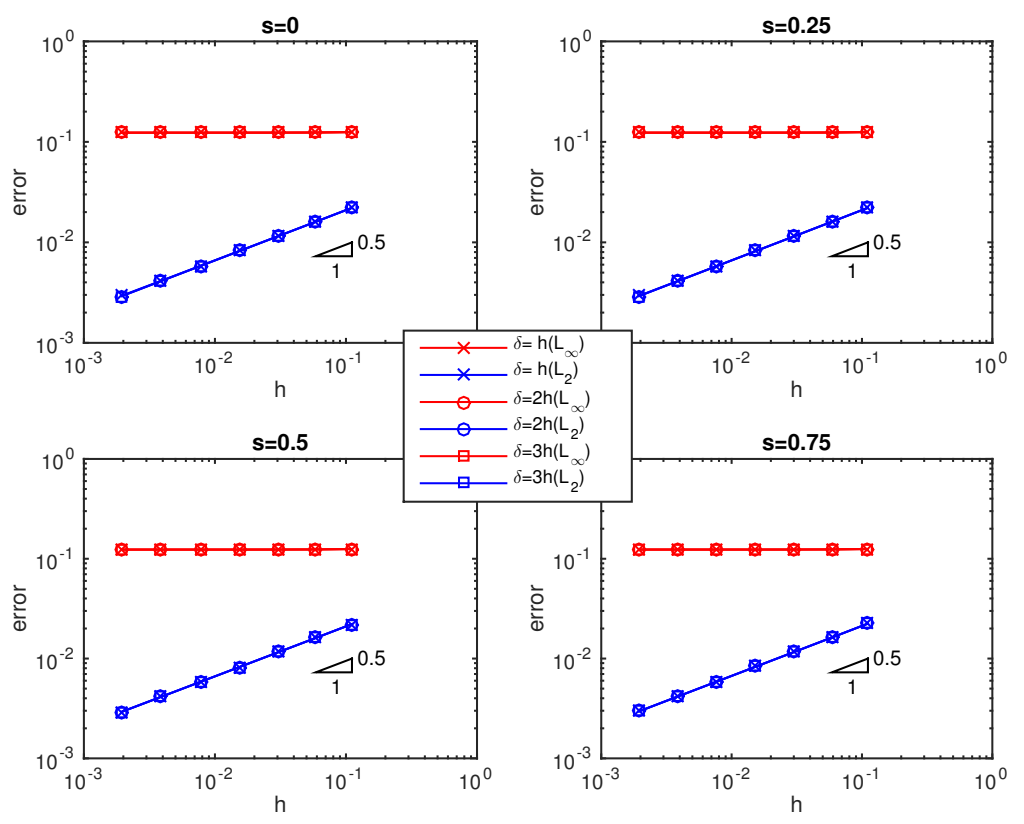

Figure 9: The same set up as for Figure 5, except that now for all grids used, no grid point coincides with the point at which the exact solution is discontinuous.

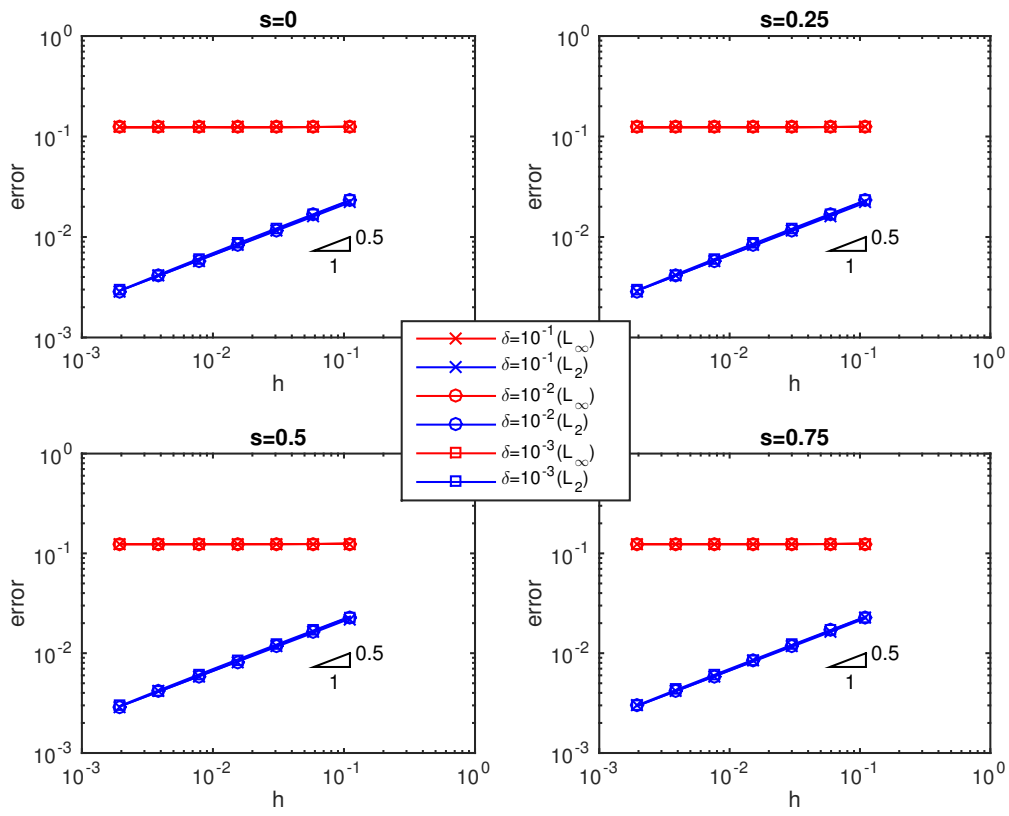

Figure 10: The same set up as for Figure 6, except that now for all grids used, no grid point coincides with the point at which the exact solution is discontinuous.

i.e., with respect to the grid size used outside the intervals containing jump discontinuities in the exact solution, the best approximation recovers the rate of convergence obtainable for smooth solutions.

The numerical results shown in Figure 13 are for discontinuous piecewise-linear approximations 


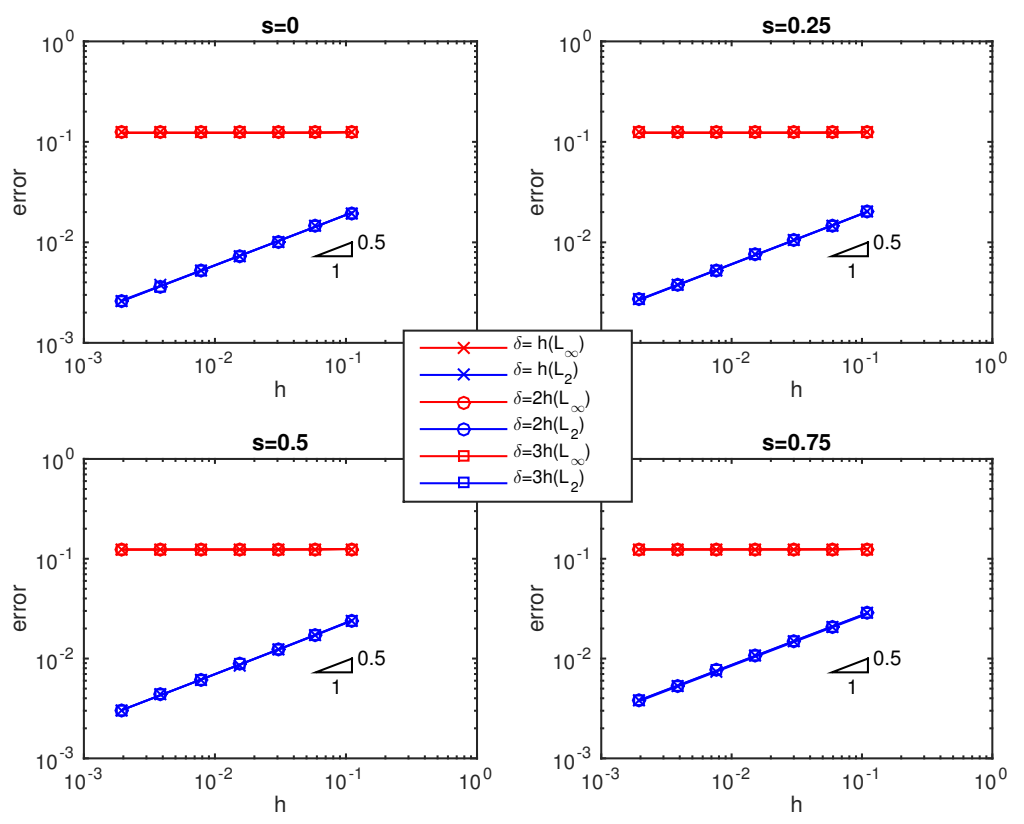

Figure 11: The same set up as for Figure 7, except that now for all grids used, no grid point coincides with the point at which the exact solution is discontinuous.

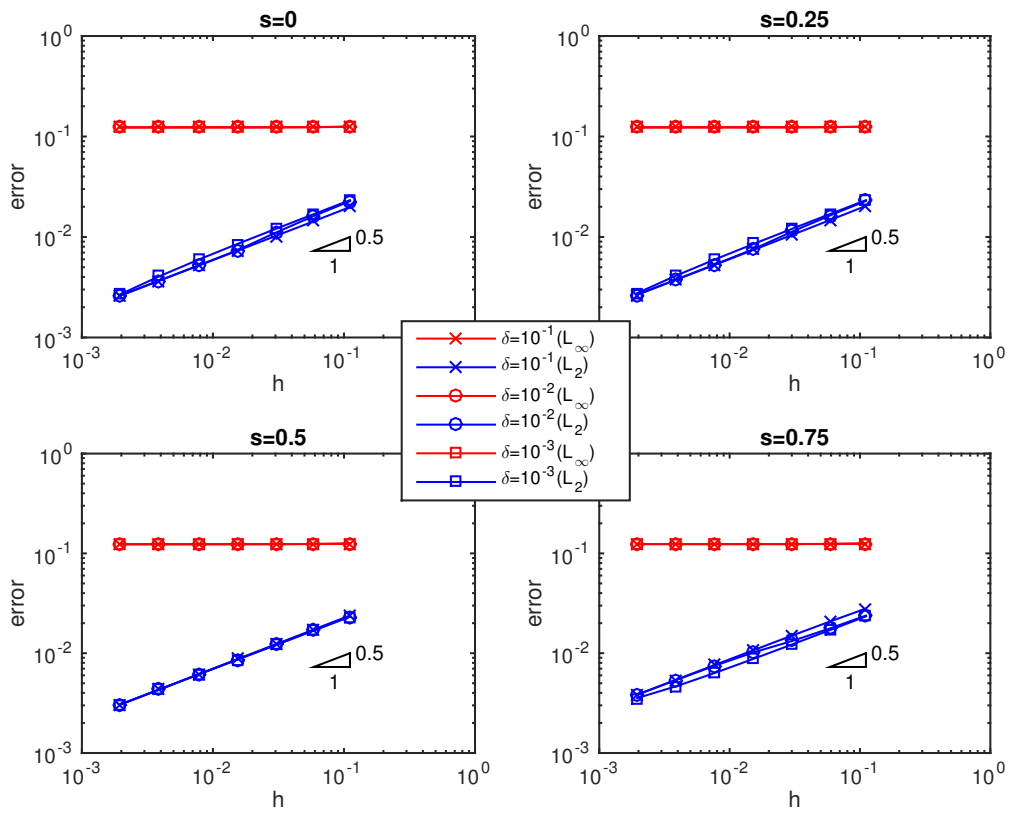

Figure 12: The same set up as for Figure 8, except that now for all grids used, no grid point coincides with the point at which the exact solution is discontinuous.

of the problem having exact solution (43) and for four values of $s \in[0,1$ ); a constant grid size $h$ is used everywhere except for the single interval containing the jump discontinuity which has a grid size $h^{4}$. For the two values of $s \in\left[0, \frac{1}{2}\right)$, we see that, even though the discontinuity in the exact solution occurs in the interior of an element, the $L^{2}$ convergence rates are the same as for smooth 
solutions and match the best approximation errors as given by (45). However, the approach of locally refining the grid does not help at all for $L^{\infty}$ errors because regardless of how small we make the element containing the jumpy discontinuity, the $L^{\infty}$ error will remain of $O(1)$. But, if we ignore the interval that containing the jump discontinuity, the errors in the best $L^{\infty}$ approximations are the same as that for smooth solutions. The same seems to hold for the finite element solution; see the top plots of Figure 14.

It should be noted that such full accuracy recovery trick fails when $s \geq 1 / 2$; see the bottom plots of Figures 13 and 14. The reason why this happened is not clear for the time being and should be further investigated.
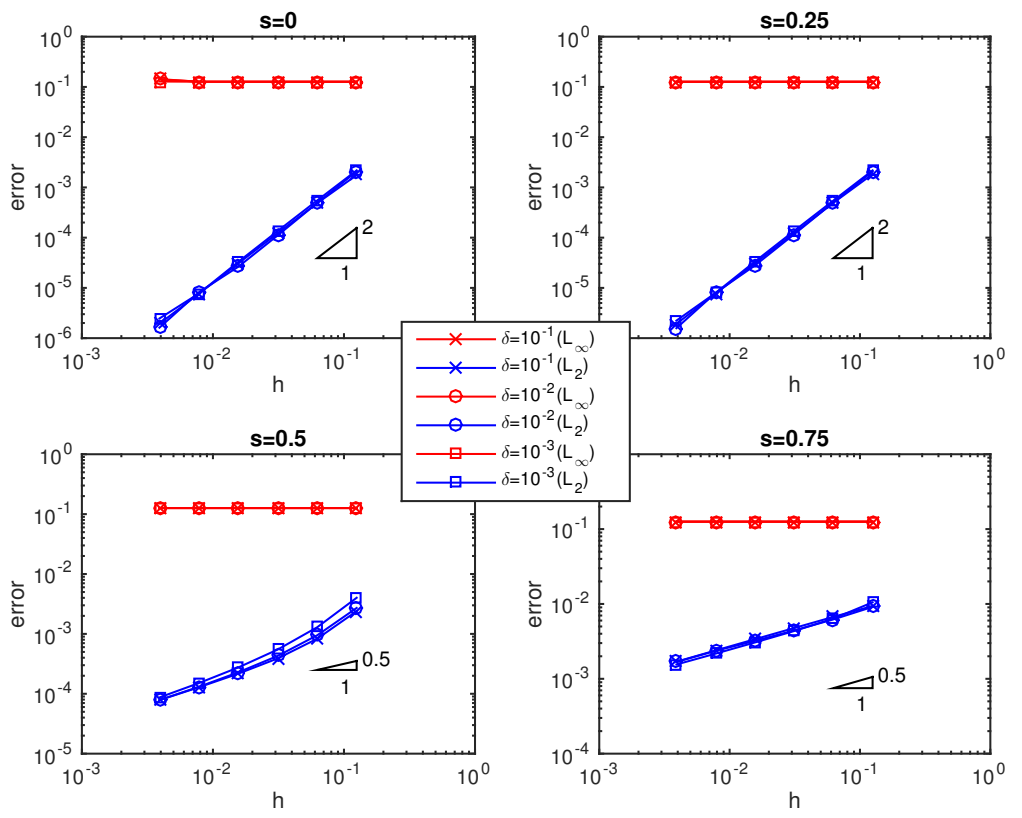

Figure 13: The same set up as for Figure 11 except that we have four values of $s \in[0,1)$ and the element containing the discontinuity in the solution has length $h^{4}$, with the remaining elements begin uniform of size $O(h)$.

Remark 12. Let $e_{d}, h_{d}$, and $h$ be described as above. If one chooses $h_{d}=h^{4}$ and $e_{m}$ and $e_{n}$ are located on either side of $e_{d}$, then, for the double integral defined by (39), the parameters $\epsilon_{1}$ in Section 5.2 and $\epsilon_{2}$ in Section 5.3 may be very small, the evaluation can proceed as in Remark 9 and 10 , respectively.

\section{Concluding remarks}

In this paper, we study a novel and efficient way to assemble the stiffness matrix in Galerkin finite element methods for ND or AD models. To compute the entries, the product rule is developed for evaluating the inner integral and then the DE-rule is used for the outer one, which make the assembly of the stiffness matrix in the finite element methods for nonlocal problems be similar to that for the classic partial differential equations and significantly reduce the quadrature points. Such approach also makes higher order finite element approximations become very simple to be implemented. 


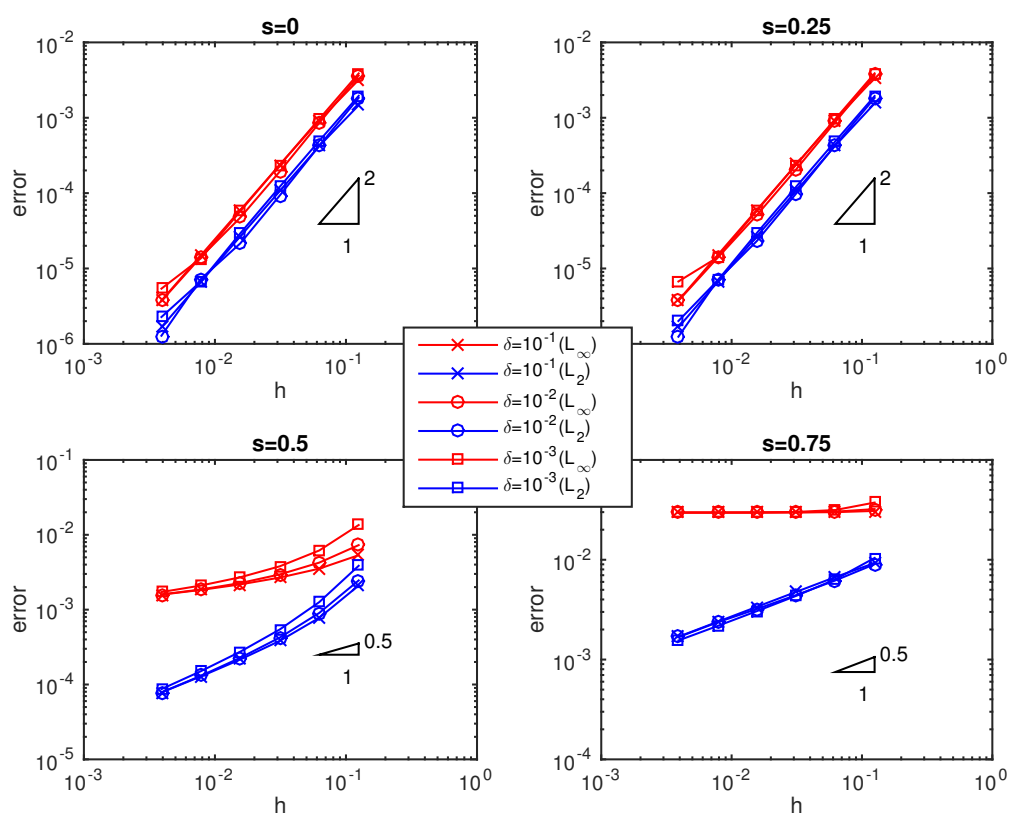

Figure 14: The same set up as for Figure 12 except that we have four values of $s \in[0,1)$ and the element containing the discontinuity in the solution is ignored, with the remaining elements begin uniform of size $O(h)$.

A possible extension of this work consists in developing quadrature rules for finite element approximations in 1D nonlocal problems with variable diffusive coefficient

$$
\mathcal{L}_{\delta} u(x)=\frac{2-2 s}{\delta^{2-2 s}} \int_{B_{\delta}(x)} \kappa(x, y) \frac{u(y)-u(x)}{|y-x|^{1+2 s}} d y
$$

where $\kappa(x, y)$ is bounded given constitutive function. Nothing is different except that the alternative form of $(22)$

$$
\hat{\theta}_{m}(x)=\int_{-1}^{1} \kappa(x, y) K(x, y)(y-x)^{m} d y
$$

should be exactly evaluated, where $K(x, y)$ denote the kernel functions discussed in Cases 1-3 of Section 3.1. In fact, we can first approximate $\kappa(x, y)$ by its Lagrange interpolant with respect to points $\left\{x_{k}\right\}_{k=1}^{n}$ denoting the zeros of the Legendre polynomials of degree $n$

$$
\kappa(x, y) \approx \sum_{k=1}^{n_{1}} \kappa\left(x, x_{k}\right) \ell_{k}(x)
$$

and then use (15) and (16), it's obvious that (46) can be approximated by

$$
\hat{\theta}_{m}(x) \approx \sum_{k=1}^{n_{1}} \frac{\lambda_{k} \kappa\left(x, x_{k}\right) P_{l}\left(x_{k}\right)}{2} \sum_{l=0}^{n_{1}-1}(2 l+1) \nu_{l, m}(x)
$$

with $\nu_{l, m}(x)=\int_{-1}^{1} \frac{P_{l}(y)(y-x)^{m}}{|y-x|^{1+2 s}} d y$. By using (20), we have

$$
\nu_{l, m}(x)=2^{-l} \sum_{k_{1}=0}^{\lfloor l / 2\rfloor}(-1)^{k_{1}}\left(\begin{array}{c}
l \\
k_{1}
\end{array}\right)\left(\begin{array}{c}
2 l-2 k_{1} \\
l
\end{array}\right) \sum_{m_{1}=0}^{l-2 k_{1}}\left(\begin{array}{c}
l-2 k_{1} \\
m_{1}
\end{array}\right) x^{l-2 k_{1}-m_{1}} \theta_{m+m_{1}}(x),
$$


where $\theta_{m}(x)$ is defined in (22). That is to say, $\hat{\theta}_{m}(x)$ defined in (46) can be approximated by certain combination of $\theta_{m}(x)$ defined in (22), which is easily implemented in practice.

Another possible extension of this work consists in developing quadrature rules for finite element approximations in $2 \mathrm{D}$ and $3 \mathrm{D}$ nonlocal problems

$$
\begin{cases}-\int_{B_{\delta}(\boldsymbol{x})} \frac{u(\boldsymbol{y})-u(\boldsymbol{x})}{r^{d+2 s}(\boldsymbol{x}, \boldsymbol{y})} d \boldsymbol{y}=f(\boldsymbol{x}) & \text { in } \Omega \subset \mathbb{R}^{d}, \\ u(\boldsymbol{x})=g(\boldsymbol{x}) & \text { on } \Omega_{\mathcal{I}} \subset \mathbb{R}^{d},\end{cases}
$$

where $d=2,3, r(\boldsymbol{x}, \boldsymbol{y})=\|\boldsymbol{x}-\boldsymbol{y}\|_{2}, f(\boldsymbol{x})$ and $g(\boldsymbol{x})$ are given functions and $\Omega_{\mathcal{I}}$ is the interaction domain. Note that $B_{\delta}(\boldsymbol{x})=\left\{\boldsymbol{y} \in \mathbb{R}^{d}:\|\boldsymbol{y}-\boldsymbol{x}\|<\delta\right\}$ may have different choices, depending on the choice of $\|\cdot\|$. For example, $B_{\delta, 2}(\boldsymbol{x})=\left\{\boldsymbol{y} \in \mathbb{R}^{d}:\|\boldsymbol{y}-\boldsymbol{x}\|_{2}<\delta\right\}$ is a circle centered at $\boldsymbol{x}$ of radius $\delta$, and $B_{\delta, \infty}(\boldsymbol{x})=\left\{\boldsymbol{y} \in \mathbb{R}^{d}:\|\boldsymbol{y}-\boldsymbol{x}\|_{\infty}<\delta\right\}$ is a square centered at $\boldsymbol{x}$ with sides of length $\delta$ in two-dimensional space.

A weak formulation of (47) is given by, for $\Omega^{\prime}=\Omega \cup \Omega_{\mathcal{I}}$,

$$
-\int_{\Omega} \int_{\Omega^{\prime} \cap B_{\delta}(\boldsymbol{x})} \frac{u(\boldsymbol{y})-u(\boldsymbol{x})}{r^{d+2 s}(\boldsymbol{x}, \boldsymbol{y})} v(\boldsymbol{x}) d \boldsymbol{y} d \boldsymbol{x}=\int_{\Omega} f v d \boldsymbol{x}, \quad d=2,3 .
$$

The difficulty of computing the entries in the stiffness matrix exists in the dimension, the irregular domain of integration and the higher-order singularity of the inner and outer integrals, as well as the kind of the mesh. The current study may be extended to construct quadrature rules on the rectangular mesh, where the accurate product rule can be proposed for the inner integrals and then the DE rules can also be used for the outer one. If the triangular/tetrahedral mesh is used, more challenges will be encountered due to the complicated intersection area of the mesh element and $B_{\delta}(\boldsymbol{x})$.

\section{References}

\section{References}

[1] S. Silling, Reformulation of elaticity theory for discontinuities and long-range forces, J. Mech. Phys. Solids 48 (2000) 175-209.

[2] S. Silling, Linearized theory of peridynamic states, J. Elast. 99 (2010) 85-111.

[3] S. Silling, M. Epton, O. Weckner, J. Xu, E. Askari, Peridynamic states and constitutive modeling, J. Elast. 88 (2007) 151-184.

[4] S. Silling, Dynamic fracture modeling with a meshfree peridynamic code, Comput. Fluid Solid Mech., Elsevier, Amsterdam (2003) 641-644.

[5] S. Silling, E. Askari, Peridynamic modeling of impact damage, J. Elast. 99 (2004) 197-205.

[6] S. Silling, E. Askari, A meshfree method based on the peridynamic model of solid mechanics, Comput. Struct. 83 (2005) 1526-1535.

[7] S. Silling, F. Bobaru, Peridynamic modeling of membranes and fibers, Int. J. Nonlinear Mech. 40 (2005) 395-409. 
[8] S. Silling, R. Lehoucq, Peridynamic theory of solid mechanics, Adv. Appl. Mech. 44 (2010) $73-168$.

[9] Q. Du, M. Gunzburger, R. Lehoucq, K. Zhou, Analysis and approximation of nonlocal diffusion problems with volume constraints, SIAM Rev. 56 (2012) 676-696.

[10] Q. Du, M. Gunzburger, R. Lehoucq, K. Zhou, A nonlocal vector calculus, nonlocal volumeconstrained problems, Math. Model. Methods Appl. Sci. 23 (2013) 493-540.

[11] B. Aksoyku, Z. Unlu, Conditioning analysis of nonlocal integral operators in fractional Sobolev spaces, SIAM J. Numer. Anal. 52 (2014) 653-677.

[12] X. Chen, M. Gunzburger, Continuous and discontinous finite element methods for a peridynamics model of mechanics, Comput. Methods Appl. Mech. Engrg. 200 (2011) 1237-1250.

[13] Q. Du, L. Ju, L. Tian, K. Zhou, A posteriori error analysis of finite element method for linear nonlocal diffusion and peridynamic models, Math. Comput. 82 (2013) 1889-1922.

[14] X. Tian, Q. Du, Analysis and comparison of different approximations to nonlocal diffusion and linear peridynamic equations, SIAM J. Numer. Anal. 51 (2013) 3458-3482.

[15] K. Zhou, Q. Du, Mathematical and numerical analysis of linear peridynamic models with nonlocal boundary conditions, SIAM J. Numer. Anal. 48 (2010) 1759-1780.

[16] H. Tian, L. Ju, Q. Du, Nonlocal convection-diffusion problems and finite element approximations, Comput. Methods Appl. Mech. Engrg. 289 (2015), 60-78.

[17] R. Metzler, J. Klafter, The random walk's guide to anomalous diffusion: A fractional dynamics approach, Phys. Rep. 393 (2000) 1-77.

[18] E. Emmrich, O. Weckner, The peridynamic equation and its spatial discretization, Math. Model. Anal. 12 (2007) 17-27.

[19] R. Maceka, S. Silling, Peridynamics via finite element analysis, Finite Elem. Anal. Des. 4 (2007) 1169-1178.

[20] H. Wang, H. Tian, A fast and faithful collocation method with efcient matrix assembly for a two-dimensional nonlocal diffusion model, Comput. Meth. Appl. Mech. Engrg. 273 (2014) $19-36$.

[21] X. Zhang, M. Gunzburger, L. Ju, Nodal-type collocation methods for hypersingular integral equations and nonlocal diffusion problems, Comput. Methods Appl. Mech. Engrg. 299 (2016), 401-420.

[22] S. Sauter, C. Schwab, Boundary element methods, Springer-Verlag Berlin Heidelberg, 2011.

[23] N. Hilber, O. Reichmann, C. Schwab, C. Winter, Computational methods for quantitative finance, Springer-Verlag Berlin Heidelberg, 2013.

[24] M. Delia, M. Gunzburger, The fractional laplacian operator on bounded domains as a special case of the nonlocal diffusion operator, Comput. Math. Appl. 66 (2013) 1245-1260. 
[25] A. Aimi, A. Carini, M. Diligenti, G. Monegato, Numerical integration schemes for evaluation of (hyper)singular integrals in 2D BEM, Comput. Mech. 22 (1998) 1-11.

[26] G. Vainikko, I. Lifanov, On the notion of the finite part of divergent integrals in integral equations, Diff. Eqn. 38 (2002) 1313-1326.

[27] J. Wu, Y. Wang, W. Li, W. Sun, Toeplitz-type approximations to the hadamard integral operator and their applications to electromagnetic cavity problems, Appl. Numer. Math. 58 (2008) 101-121.

[28] W. Sun, J. Wu, Interpolatory quadrature rules for hadamard finite-part integrals and their superconvergence, IMA. J. Numer. Anal. 28 (2009) 580-597.

[29] G. Tsamasphyros, G. Dimou, Gauss quadrature rules for finite part integrals, Int. J. Numer. Methods Engrg. 30 (1990) 13-26.

[30] G. Monegato, Numerical evaluation of hypersingular integrals, J. Comput. Appl. Math. 50 (1994) 9-31.

[31] C. Hui, D. Shia, Evaluations of hypersingular integrals using Gaussian quadrature, Int. J. Numer. Methods Engrg. 44 (1999) 205-214.

[32] J. Wu, W. Sun, The superconvergence of newton-cotes rules for the hadamard finite-part integral on an interval, Numer. Math. 109 (2008) 143-165.

[33] D. Elliott, E. Venturino, Sigmoidal transformations and the euler-maclaurin expansion for evaluating certain hadamard finite-part integrals, Numer. Math. 77 (1997) 453-465.

[34] U. Choi, S. Kim, B. Yun, Improvement of the asymptotic behaviour of the euler-maclaurin formula for cauchy principal value and hadamard finite-part integrals, Int. J. Numer. Methods Engrg. 61 (2004) 496-513.

[35] P. Kim, U. Jin, Two trigonometric quadrature formulae for evaluating hypersingular integral, Int. J. Numer. Methods Engrg. 56 (2003) 469-486.

[36] G. Szegö, Orthogonal polynomials (4th Ed.), Providence, RI, Amer. Math. Soc., 1975.

[37] D. Elliot, D. Paget, Product-integration rules and their occurrence, BIT 16 (1976) 32-40.

[38] H. Takahasi, M. Mori, The double exponential transformation in numerical analysis, J. Comput. Appl. Math. 127 (2001) 287-296. 